\title{
Readiness Discharge for Spontaneous Initiation of Walking in Crayfish
}

\author{
Katsushi Kagaya ${ }^{1}$ and Masakazu Takahata ${ }^{1,2}$ \\ ${ }^{1}$ Division of Biological Sciences, Graduate School of Science, and ${ }^{2}$ Department of Life Sciences, Faculty of Science, Hokkaido University, Sapporo 060-0810, \\ Japan
}

Animals initiate behavior not only reflexively but also spontaneously in the absence of external stimuli. In vertebrates, electrophysiological data on the neuronal activity associated with the self-initiated voluntary behavior have accumulated extensively. In invertebrates, however, little is known about the neuronal basis of the spontaneous initiation of behavior. We investigated the spike activity of brain neurons at the time of spontaneous initiation of walking in the crayfish Procambarus clarkii and found neuronal signals indicative of readiness or preparatory activities in the vertebrate brain that precede the onset of voluntary actions. Those readiness discharge neurons became active $>1 \mathrm{~s}$ before the initiation of walking regardless of stepping direction. They remained inactive at the onset of mechanical stimulus-evoked walking in which other descending units were recruited. These results suggest that the parallel descending mechanisms from the brain separately subserve the spontaneous and stimulus-evoked walking. Electrical stimulation of these different classes of neurons caused different types of walking. In addition, we found other descending units that represented different aspects of walking, including those units that showed a sustained activity increase throughout the walking bout depending on its stepping direction, as well as one veto unit for canceling out the output effect of the readiness discharge and three termination units for stopping the walking behavior. These findings suggest that the descending activities are modularized in parallel for spontaneous initiation, continuation, and termination of walking, constituting a sequentially hierarchical control.

\section{Introduction}

The readiness potential was first found by Kornhuber and Deecke (1965) to precede voluntary acts by some hundred milliseconds in humans. It still remains open whether the potential itself represents the volition or the free will of the subject (Gomes, 1999), but it is an established fact that neuronal activities that precede the onset of self-initiated behavior can be recorded in specific regions of the primate brain, apparently corresponding to the readiness potential (Schultz et al., 1997; Purzner et al., 2007). In vertebrates, electrophysiological data on the neuronal activity related to the self-initiated voluntary behavior have accumulated extensively (Schultz, 2000; Schall, 2001; Maimon and Assad, 2006; Tanji and Hoshi, 2008). In contrast, there are only a few lines of physiological evidence for such self-initiated behavior in invertebrates (Brodfuehrer and Thorogood, 2001; Garcia-Perez et al., 2007), although they have been exploited for the investigation of neuronal mechanisms subserving a variety of stimulusevoked behavior (Clarac and Pearlstein, 2007).

The central pattern-generating systems have been well characterized in many invertebrates for locomotor behavior (Robertson,

Received 0ct. 1, 2009; revised Nov. 10, 2009; accepted Nov. $27,2009$.

This work was supported by Grants-in-Aid for Scientific Research from the Ministry of Education, Culture, Sports, Science and Technology, Japan (20370028 and 18657025). We are grateful to Drs. N. Hama and A. Takashima for their suggestions and criticisms in the course of this study. We also thank anonymous reviewers for their constructive criticisms and suggestions.

Correspondence should be addressed to Katsushi Kagaya, Division of Biological Sciences, Graduate School of Science, Hokkaido University, Sapporo 060-0810, Japan. E-mail: kagaya@sci.hokudai.ac.jp.

D01:10.1523/JNEUROSCI.4885-09.2010

Copyright $\odot 2010$ the authors $\quad 0270-6474 / 10 / 301348-15 \$ 15.00 / 0$
1986; Ramirez and Pearson, 1988; Burrows, 1996; Pearson and Ramirez, 1997; Kristan and Gillette, 2007; Clarac, 2008). In Drosophila, the functional relationship between locomotor activity and brain structure has been well described (Martin et al., 1998, 1999a,b). Specific aspects of spontaneous locomotor behavior have been related to specific regions in the Drosophila brain (Martin, 2003, 2004). However, it remains unknown what kind of neuronal activities connect the animal's decision to walk with the stepping pattern generator in the thoracic ganglia. Furthermore, behavioral analyses of temporal patterns of flight behavior in Drosophila have suggested that a nonlinear dynamic initiator of the behavior is implemented in the CNS (Maye et al., 2007), but its neuronal basis remains unknown.

In crayfish, Bowerman and Larimer (1974a,b) found that some fibers in the circumesophageal connectives could produce a whole or part of walking behavior in either forward or backward direction on electrical stimulation. They reported 121 command fibers for different behaviors including walking. The rhythmic movements of walking legs for stepping themselves are produced by the patterngenerating circuits located in the thoracic ganglia (Chrachri and Clarac, 1990). There is no information, however, how and which of the command fibers are activated to drive the pattern generator when the animal initiates walking behavior spontaneously.

Our previous study using electromyographic (EMG) techniques revealed that all leg muscles were tonically activated before the onset of rhythmical stepping movements by approximately one-half of a second when the animal initiated walking spontaneously. This preactivation time was significantly shorter in the mechanical stimulus-evoked walking (Chikamoto et al., 2008). 
The question we addressed here is what kind of neural activity in the crayfish brain causes the long-lasting preactivation of leg muscles before spontaneous initiation of walking. We report that spontaneous initiation of walking in crayfish is represented by a specific spike activity of descending neurons, which we termed readiness discharge, preceding the onset of stepping movements $>1 \mathrm{~s}$.

\section{Materials and Methods}

Animals and preparation. We performed experiments on adult crayfish Procambarus clarkii of both sexes ranging from 8 to $12 \mathrm{~cm}$ in body length. They were kept in laboratory tanks maintained under a $12 \mathrm{~h}$ light/dark cycle. To keep the motivational state of experimental animals as constant as possible, they were given nothing to eat $2-3 \mathrm{~d}$ before the physiological recording. Both chelipeds were occasionally cut off before experiments, but we did not observe any noticeable difference in the performance of walking between intact and operated animals. A steel nut was glued on the dorsal surface of carapace so that an animal could be held on a newly developed treadmill apparatus (see Fig. 1). For electromyographic recording, a pair of wire electrodes (Teflon-coated silver wires of diameter $125 \mu \mathrm{m}$ ) was implanted to the mero-carpopodite flexor muscle (Chikamoto et al., 2008) in the second walking leg on either side. A small portion of the dorsal carapace was removed to expose the circumesophageal commissures. One of the commissures was partly split and cut off so that the multiunit extracellular recording could be made from the descending cut end with a suction electrode. Typically, a bundle contained 2-13 functional units relevant to locomotor behavior. The approximate location of the recorded bundle was mapped on the standard cross-sectional diagram of Wiersma (1958) for the circumesophageal commissure. Note that the commissure on the other side was always kept intact. The surgery was kept to the minimal to maximize the longevity of the preparation: some animals behaved just like an intact animal for $>240 \mathrm{~min}$. Those animals that showed apparently abnormal inactivity or those that did not show spontaneous walking were discarded before experiment.

Treadmill system. The experimental setup is illustrated in Figure 1. The animal was placed on a sphere-type treadmill in such a way that it could freely perform locomotor behavior. The lever, to which the animal body was fixed by its cephalothorax and the electrode holder was attached together with a micromanipulator, could move around a pivot as the animal stood up and down in the locomotor behavior. The weight of the lever was canceled by a counterweight placed on the other end of the lever over the pivot. The whole system including the animal was placed in the air. To record the direction and velocity of the sphere attributable to leg movements of the behaving crayfish, two optical mice were placed on the basal and side planes for tracking the forward/backward translation and clockwise/counterclockwise turning, respectively. The information from these devices was sampled at the frequency of $60 \mathrm{~Hz}$ and fed in two computers in parallel to record the animal movement/position on electronic devices and to visualize it on the screen of a computer display in real time.

Reconstruction of walking behavior. The walking behavior of crayfish on the treadmill was quantified by digitizing the three rotation vectors that represented the forward, backward, and turning behavior. The digitized data were associated with a train of pulse signals that were also fed to one channel of the neurophysiological recording system (see below) to synchronize behavioral data with neural unit activities. From those vectors, we reconstructed the walking behavior of crayfish assuming that the longitudinal rotation of the sphere indicated the forward and backward movement, whereas the horizontal rotation indicated the turning movement around the vertical axis.

To analyze the spike activity relating to the forward and backward walking, we categorized walking behavior into four walking types, that is, forward and backward walking and clockwise and counterclockwise turning. Although curved forward and backward walking patterns (Yamane and Takahata, 2002) were also found on the treadmill, we selected for the present analyses of directional unit activities only the data series of straight forward and backward walking: if the animal position measured $10 \mathrm{~s}$ after the behavioral onset of walking was in the direction within $\pm 45^{\circ}$ ahead or backward, we categorized the behavior into forward or backward walking, respectively. The walking in other di- rections was excluded from the analysis of the directional selectivity of unit activities (see Fig. 5).

EMG analysis. For determining the time of behavioral onset of walking, we applied the minimum Akaike information criterion (AIC) procedure to the EMG record. This procedure was originally applied to the estimation of the arrival time of an earthquake signal in seismology. Theoretical details and computational algorithm of the procedure are provided by Kitagawa et al. (2001). Although the EMG activity was nonstationary because of dynamic changes over a long time span, it could be considered as stationary for a sufficiently short period. For this reason, we applied this procedure in the same way as in the seismological procedure. Briefly, we chose one or more $1 \mathrm{~s}$ block (time series of 1000 points under the sampling rate of $1 \mathrm{kHz}$ ) to apply the procedure. The block containing the time of behavioral onset could be divided into two blocks. To determine the dividing point, the block was provisionally divided into two blocks. Each block was fitted by an autoregressive model (highest autoregressive orders of 20) and the goodness of fit for each model was measured by the corresponding AIC. The dividing point was moved step by step (moved forward point by point), and if the sum of each AIC got the minimum value, the dividing point was judged to provide the best model. The time of the dividing point gave the time of behavioral onset.

It should be noted here that the real onset time of walking behavior as the visually recognizable movements is preceded by an increase in the EMG activity of walking legs by approximately one-half of a second in the spontaneously initiated walking. We analyzed this phenomenon quantitatively in our preceding paper (Chikamoto et al., 2008). Therefore, the onset time of EMG activity increase is not precisely the same as the behavioral onset time. We used the term "behavioral onset," however, for the time of EMG activity increase throughout this study to avoid unnecessary complication of our description.

Multiunit recording. We recorded the spike activity of descending neurons from the cut end of the circumesophageal commissure with a suction electrode simultaneously with the electromyographic recording from a walking leg. Those physiological data were digitized at the sampling rate of $20 \mathrm{kHz}$ for the descending activity and $1 \mathrm{kHz}$ for the electromyographic recording using ADInstruments PowerLab ML785 and stored on a personal computer. The recording could be maintained $\sim 1 \mathrm{~h}$ : the first $30 \mathrm{~min}$ were used for recording only the spontaneous walking and next $30 \mathrm{~min}$ for recording both the spontaneous and mechanical stimulus-evoked walking. Since the unit amplitude slightly changed over time during the recording session, we separately analyzed the first and second halves of the recorded data. All spikes were first sorted depending on their amplitude using a spike analysis software (Spike2; CED; version 6.0). We then mapped each waveform of descending units into the threedimensional space constructed from principal component analyses, and selected 2-13 clusters manually by the ellipsoidal selector in the tool of Spike2. This procedure allowed us to isolate specific units from the multiunit recording data. The timings of each unit spike discharges were obtained and exported to another homemade computer program for compiling the spike raster display and histogram.

Stimulation. Crayfish can be made to initiate locomotor behavior reflexively by stimulating the animal body either mechanically or chemically in the underwater condition (Chikamoto et al., 2008). In the present study, however, we adopted only the mechanosensory stimuli since the treadmill system, using a Styrofoam sphere, could not be used in the underwater condition. To evoke walking by mechanical stimulation, the animal was stimulated either manually by a soft paintbrush applied to the telson or electrically (pulses of $5 \mathrm{~ms}$ in duration and $100 \mathrm{~Hz}$ in frequency) by a pair of silver wire electrodes ( $125 \mu \mathrm{m}$ in diameter and $5 \mathrm{~mm}$ in length) implanted into the telson from the lateral side so that they were arranged in parallel ( $1 \mathrm{~mm}$ distance) and perpendicular to the longitudinal body axis to stimulate mechanosensory afferents (supplemental Fig. 4, available at www.jneurosci.org as supplemental material). In manual stimulation, its timing was determined by the stimulus artifact attributable to a capacitative noise in the EMG record. In either mechanical or electrical stimulation, the stimulus intensity was kept low so as not to elicit escape tail flip. The walking response followed the stimulation with a delay of approximately one-half of a second (mean \pm SD, $591 \pm 265 \mathrm{~ms}$ ). 
To investigate how the spike activities of descending units are causally related to walking behavior, we electrically stimulated one of them and tested whether the stimulation had any effect on the animal behavior. Since it was difficult in practice to physically isolate one single descending unit, we split off a small nerve bundle containing a putative functional unit with relatively large spike amplitude from the circumesophageal commissure on either side. To stimulate the fibers we intended to, in stimulation experiments, the isolated bundles were smaller than the bundles that were used only for recording experiments. The commissure on the other side remained intact. The bundle was then severed by a pair of microscissors to yield a rostral cut end from which descending unit activities were recorded extracellularly, and a caudal cut end to which a suction electrode was applied to stimulate the functional unit electrically. Since the commissure remained intact on one side, the animal could normally show spontaneous and mechanical stimulus-evoked walking behavior. After confirming that the bundle contained either of the readiness discharge unit, the mechanical stimulus-evoked unit, or the termination unit, we stimulated the bundle at the caudal cut end of the commissure to analyze its effects on the animal behavior using EMG and treadmill recordings. For stimulation, a train of pulses with the duration of $0.1 \mathrm{~ms}$ and the frequency of $50 \mathrm{~Hz}$ were applied to the electrode for $4 \mathrm{~s}$ or longer up to $16 \mathrm{~s}$. The pulse intensity, controlled in the constantcurrent mode, was gradually increased until the animal consistently displayed any unambiguous behavior. The maximal stimulus intensity varied from 20 to $50 \mu \mathrm{A}$ depending on the preparation. Our histological study demonstrated that the part of the commissure ventral to the medial giant contains no particular large fibers (our unpublished data). It thus followed that the unit with the largest spike amplitude in the bundle from that part of the commissure was the one that was located electrically nearest to the electrode. We assumed, therefore, that it was the readiness discharge unit showing the largest spike amplitude that was first activated when the stimulus intensity was increased gradually.

Analysis of spike activity. The selective activity of each unit before walking that was initiated spontaneously or reflexively (see Fig. 4C), and in the forward or backward direction (see Fig. 5C), was quantified by comparing the spike discharge rate between at rest and at the onset of each behavior by performing generalized linear modeling (Venables and Ripley, 2002). We first defined two periods for data analysis: the control period ( 1 or $2 \mathrm{~s}$ duration, $20 \mathrm{~s}$ before the behavioral onset) and the test period ( 1 or $2 \mathrm{~s}$ duration, 1 or $2 \mathrm{~s}$ before the onset) for each bout of walking. Spike discharge numbers were counted for each period. An experimental animal usually displayed $8-12$ bouts of spontaneous walking, and the same number of data set (i.e., control and test) was obtained from the same animal with an interval of $30-60 \mathrm{~s}$, which suggested that each data set was independent from each other. Since the data were discrete and non-negative, both the control and test populations were assumed to follow Poisson distribution. In the current analysis, we tested the possibility that the spike discharge number varied between the control and test period as well as between spontaneous/reflexive or forward/ backward walking. Therefore, using logarithm as the canonical link function for Poisson distribution in the modeling, the mean spike discharge number $(f)$ for a population was written as follows:

$$
\log f=\beta_{0}+\beta_{1} \times(\text { time point })+\beta_{2} \times(\text { behavioral category })
$$

or

$$
f=\exp \left(\beta_{0}+\beta_{1} \times(\text { time point })+\beta_{2} \times(\text { behavioral category })\right),
$$

where time point and behavioral category are factorial explanatory variables. After the maximum-likelihood estimation, the parameter values $\left(\beta_{0}, \beta_{1}, \beta_{2}\right)$ that maximized the log likelihood of Equation 2 were obtained by using the $\operatorname{glm}($ ) function of the statistical package $\mathrm{R}$ (version 2.9.1; http://www.r-project.org).

We first tested the statistical model II in which $\beta_{2}=0$ in Equation 2 against the model III in which $\beta_{1}=\beta_{2}=0$ to sort out time-dependent units (i.e., the units that showed a greater number of spikes in the test period than in the control period). Then the time-dependent units were classified into six types of behavior-dependent units by testing model I in which $\beta_{1} \neq 0$ and $\beta_{2} \neq 0$ in Equation 2 against model II. The model selection was based on AIC, which can be expressed as follows: AIC $=-2 \log L+2 k$, where $L$ is the maximum log-likelihood and $k$ is the number of parameters involved in the model (Akaike, 1974). When model I was selected over II in the spike activity of a unit, it was judged to be a spontaneous/reflexive-selective (see Fig. $4 C$ ) or forward/backward-selective (see Fig. 5C) unit depending on the behavioral category tested. Calculation of the AIC value was performed by the same $\operatorname{glm}()$ function of the R package.

The results of model selection for each unit are plotted against the ordinate for spontaneous and the abscissa for evoked walking in Figure $4 C$ and against the ordinate for forward and the abscissa for backward walking in Figure 5C. Data points shown with filled circles and open triangles in Figure $4 C$ indicate the units that were judged to be selectively activated before spontaneous and mechanical stimulus-evoked walking, respectively, whereas those in Figure $5 C$ indicate the forward- and backward-selective units. In both figures, the cross symbol represents nonspecific unit regarding the type of walking behavior (see Fig. $4 C$ ) and the walking direction (see Fig. $5 C$ ).

The selectivity of each unit could be quantified by the distance of each point from the diagonal line, $y$ (ordinate) $=x$ (abscissa), since the point should be on the line when it showed the same increase or decrease from the resting discharge rate in the two actions represented by the orthogonal coordinate axes. This distance was measured successively over time in each unit, by shifting the $1 \mathrm{~s}$ time bin for comparison by $0.5 \mathrm{~s}$ from the resting bin (i.e., $20 \mathrm{~s}$ before the onset of walking) to rightward or forward bins ending at the one just $0.5 \mathrm{~s}$ before the behavioral onset (see Fig. $4 D$ ).

\section{Results}

Together, 32 animals were used in this study, 12 for comparing the unit activity between spontaneous and mechanical stimulusevoked walking, 10 for comparison between forward and backward walking, 3 for electrical stimulation of descending units, and 1 for excision experiments of the commissure. Some animals were used for more than one experiment.

\section{Descending activities preceding the behavioral onset of spontaneously initiated walking}

Fifty-eight among 180 descending units from 21 animals exhibited any form of change in the spike activity before the onset of spontaneous walking. Stepping velocities and courses of the animal were reconstructed from the three rotation vectors of the trackball and were associated with the neurophysiological data (Fig. 1). Typical recordings from the ventromedial part of the commissure (Fig. $2 \mathrm{~B}$ ) are illustrated in Figure 2C. Intracellular staining from the cut end of the recorded bundle revealed that all stained descending units had their cell bodies in the ventral paired anterior cluster on the side opposite to the recorded axons, projecting their dendrites to the anterior and posterior medial protocerebral neuropil (supplemental Fig. 1, available at www. jneurosci.org as supplemental material) (Sandeman et al., 1992; Tautz and Müller-Tautz, 1983). Using a spike analysis software package, four descending units were discriminated that showed activity changes when the animal initiated walking spontaneously (Fig. 2C). One unit among them, designated unit 1 in this study, characteristically showed a significant increase in the firing rate before the onset of walking by $\sim 18 \mathrm{~s}$ and returned to the original activity level when the animal started walking behavior (Fig. $2 D$ ). The animal walked forward three times in this recording session (Fig. 1B). Since no external sensory stimulus was applied, this activity change was brought about endogenously. It would take some time for the motor command to travel from the brain to the thoracic ganglia, where the leg motor system is located, and fi- 
A

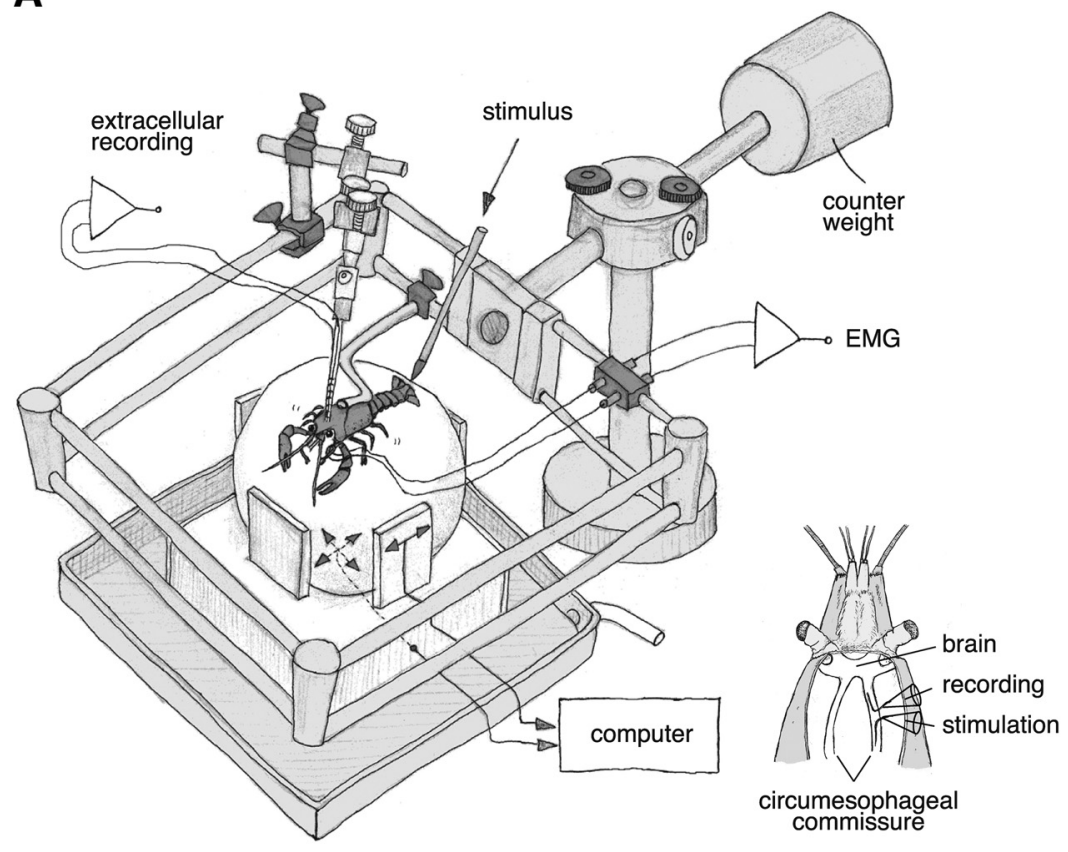

B
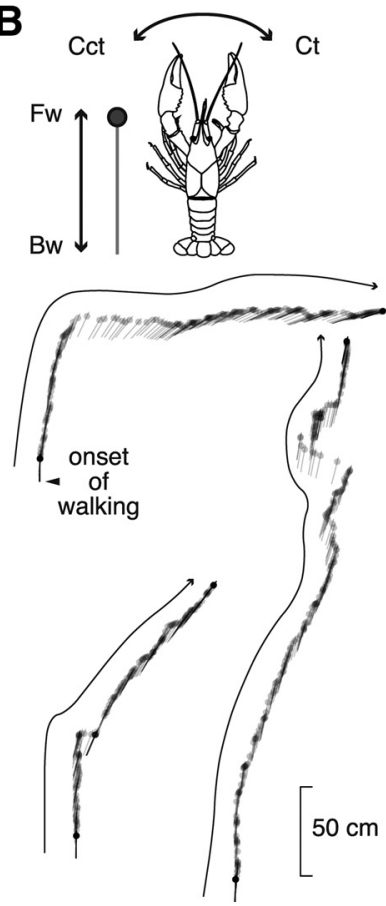

Figure 1. A spherical treadmill system used for extracellular recording from the brain of crayfish during walking and its quantification. $A$, An overview of the system. Two optical mice were placed on the basal and side planes. Those mice translated the three rotation vectors of the sphere movement caused by the spontaneous or mechanical stimulus-evoked walking of crayfish. The animal could stand up and down freely on the sphere together with the electrode and its holder whose weight was canceled out by the counter weight. A tactile stimulus was given to the telson manually with a soft brush. For defining the behavioral onset time, we made electromyographic recordings from the mero-carpopodite flexor muscle in the second walking leg on either side (Chikamoto et al., 2008). The inset shows a schematic dorsal view of the recording and stimulation with suction electrodes from the cut ends of small bundles isolated from the circumesophageal commissure. $\boldsymbol{B}$, Behavioral data of the animal during the recording session. The animal showed forward walking three times. The filled circle indicates the animal head, and the straight line indicates the body axis orientation. The trajectory consists of schematic representation of the animal body sampled every one-sixth s. Fw, Forward walking; Bw, backward walking; Ct, clockwise turning; Cct, counterclockwise turning.

nally to excite muscle fibers for contraction, but the whole required time should be much shorter than the period of preonset activity of the unit. The unit 1 activity thus represents some form of internal processes in the brain that occurs before spontaneous initiation of walking. In contrast, the other three units $(2,3,4)$ did not show any activity change before the initiation of spontaneous walking, but decreased (Fig. $2 E, F$, units 2 and 3, respectively) or increased (Fig. $2 G$, unit 4 ) their firing rate concurrently with the onset of spontaneously initiated walking. The decreased and increased activities lasted as long as the animal continued walking (Fig. 2E-G).

\section{Readiness discharges recorded consistently from different animals}

Spike activities of five different units from different animals are illustrated in Figure $3 A-E$, left, with all behavioral patterns each animal displayed during the recording session (right). It is noted that the activity profile is different from unit to unit, but the most important feature of those five units in Figure 3 was that they did not relate to any specific form of walking (e.g., straight forward or curved backward walking) but to the general locomotor behavior itself: they increased firing rate before the onset of walking whether the animal walked forward or backward or showed turning behavior. Our finding suggested that these descending activities might represent not how to nor where to walk but when to walk in the CNS of crayfish. Therefore, we termed this descending activity that occurs before spontaneous initiation of locomotor behavior as readiness discharge.

The readiness discharge unit shown in Figure $3 A$ began to increase the spike activity $>10 \mathrm{~s}$ before the behavioral onset as judged on the spike activity histogram compiled from the isolated spike trains that preceded six bouts of spontaneously initiated walking. The latency, together with that for the unit shown in Figure $2 D$, seems to be excessive for initiation of a specific behavioral act. It is noted, however, that the temporal profile in the spike train is different among the three (Fig. $2 D$, top trace) or six (Fig. 3A, top trace) bouts of walking. Such variability suggests that the readiness activity in the crayfish brain is represented by not a single unit but a sum of many readiness discharge units that shows different time courses of spike activity changes.

The readiness discharge was almost always associated with the spontaneous initiation of walking (Figs. 2D, 3). In one animal, however, we found that the readiness discharge was not associated with behavior when it was immediately followed by spike activities of another unit. The readiness discharge was consistently associated with walking whenever it was not followed by this unit activity. Since the unit appeared to cancel out the output of readiness discharge, we designated it "veto unit" in this study. Details on this unit are described in the supplemental material (available at www.jneurosci.org).

\section{Parallel descending pathways for initiating spontaneous and} mechanical stimulus-evoked walking

We next examined whether the readiness discharge was unique to the walking initiated spontaneously since the animal could also initiate walking in response to mechanical stimulation of the body. The recordings shown in Figure $4, A$ and $B$, contain two discernible descending units (arrowheads). They exhibited an increased firing rate in different conditions. One unit (black arrowhead) showed an increase in the spike discharge rate before 


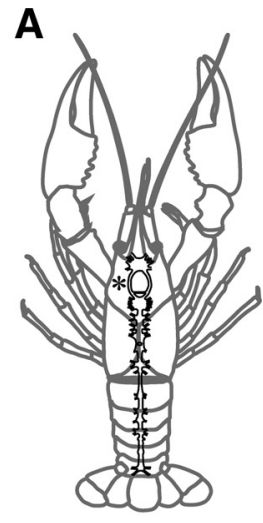

B

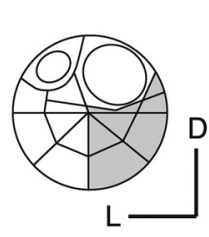

E

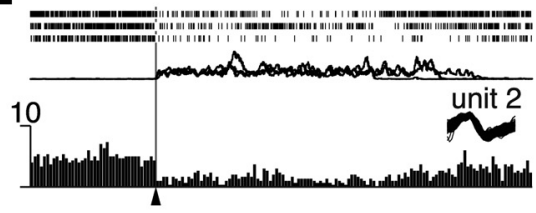

C

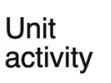

Descending activity

Leg EMG

Walking

speed

\section{D}

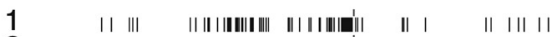

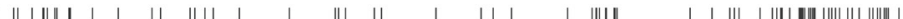

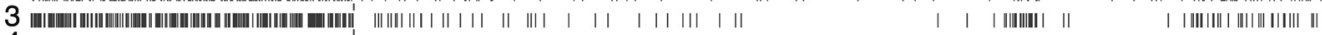

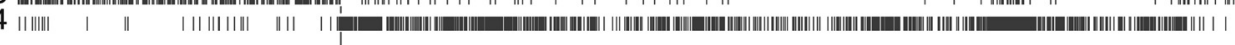

\section{unit 14}

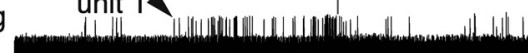

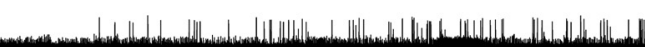

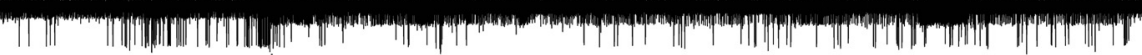

onset of walking

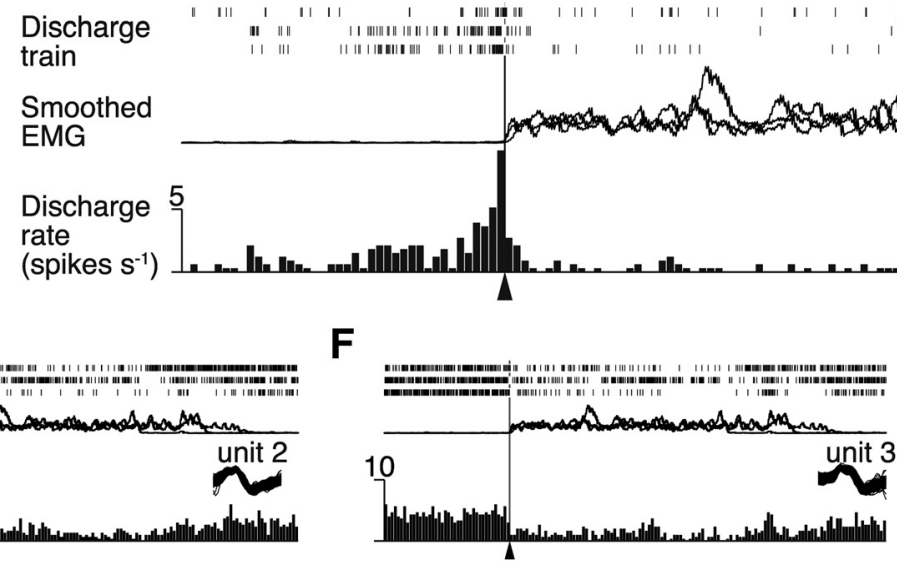

G

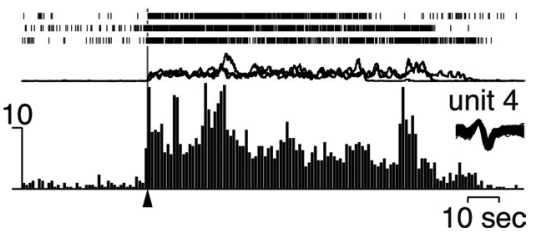

Figure 2. Descending unit activities recorded from a crayfish walking freely on a spherical treadmill. $A$, CNS of crayfish. $B$, Location on the cross-sectional map of Wiersma (1958) of the small bundle from which the recording was made in the circumesophageal commissure at the site shown with an asterisk in $\boldsymbol{A}$. C, Four descending unit activities (1-4) isolated from the bundle recording by a spike analysis software. In the top panel are shown their spike trains. The extracellular recording is shown in the next panel, followed by an EMG and the record of treadmill movements. $\boldsymbol{D}$, Activity profiles of unit 1. All obtained recordings are shown with rasters of spike trains on the top. Smoothed EMGs are superimposed below. The three traces of the responses of unit 1 were recorded during the three walking bouts in Figure $1 B$. At the bottom is shown a perimovement time histogram with the time bin of $1 \mathrm{~s}$. They are aligned to the behavioral onset time of walking. Activity of unit 1 (arrowhead) showed a significant increase before the onset of walking (vertical line). $\boldsymbol{E}-\mathbf{G}$, Activity profiles of three descending units (2-4) with the same convention as in $\boldsymbol{D}$.

the spontaneously initiated walking (Fig. 4A), but no increased activity before the initiation of mechanical stimulus-evoked walking (Fig. 4B). However, another unit (gray arrowhead) showed no activity change before the spontaneously initiated walking (Fig. 4A), yet showed an increased activity concurrently with the initiation of mechanical stimulus-evoked walking (Fig. $4 B$ ). It first appeared to be perplexing that neural signals descended from the brain to thoracic ganglia when the animal started to walk in response to mechanical stimulation of the telson. But both types of walking, spontaneous and mechanical stimulus-evoked, were found to require descending signals for generating normal movement patterns (supplemental Fig. 4, available at www.jneurosci.org as supplemental material), although it remains unknown whether the units found here represent the requirement.

The activity change of all 81 units obtained from 12 animals is illustrated in Figure $4 C$, where the change from the resting discharge rate in spontaneous walking is plotted against the ordinate, whereas that in evoked walking is plotted against the abscissa. On the left of Figure $4 C$, we compared the spike discharge rate between the premovement period of $1 \mathrm{~s}$ before the behavioral onset and the resting period of $1 \mathrm{~s}$ preceding the onset by $20 \mathrm{~s}$. On the right of Figure $4 C$, a similar comparison is made between the resting period and the $1 \mathrm{~s}$ period spanning evenly over the behavioral onset time. This latter comparison allowed us to attain a closer quantification for the mechanical stimulusevoked walking since the unit activity related to this type of walking preceded its onset by only $\sim 100 \mathrm{~ms}$ and lasted for a while after the walking was initiated. In the preonset period, 10 descending units showed selective activity changes for the spontaneously initiated walking and 33 units for the evoked walking. In the perionset period, 8 and another 42 units were selectively activated in the spontaneously initiated and mechanical stimulus-evoked walking, respectively. The temporal change in the selective activity of each unit was examined by successively shifting the $1 \mathrm{~s}$ analysis window by $500 \mathrm{~ms}$. The results are superimposed in Figure $4 D$ for all units. The unit that showed the most remarkable selectivity for spontaneous walking is drawn with a bold line. This and some other units increased their spike activity $>2 \mathrm{~s}$ before the onset of spontaneous walking, but showed no activity change before the onset of mechanical stimulus-evoked walking. Their activity thus exhibited clear selectivity in the premovement period, indicating that the readiness discharge was unique to the spontaneously initiated walking.

Descending activities for spontaneous forward and backward walking

Approximately one-third of the units examined in this study (25 of 72; the maximal recruited number of nonselective units in Fig. $5 D$ ) related to no specific type of walking, but rather to the 


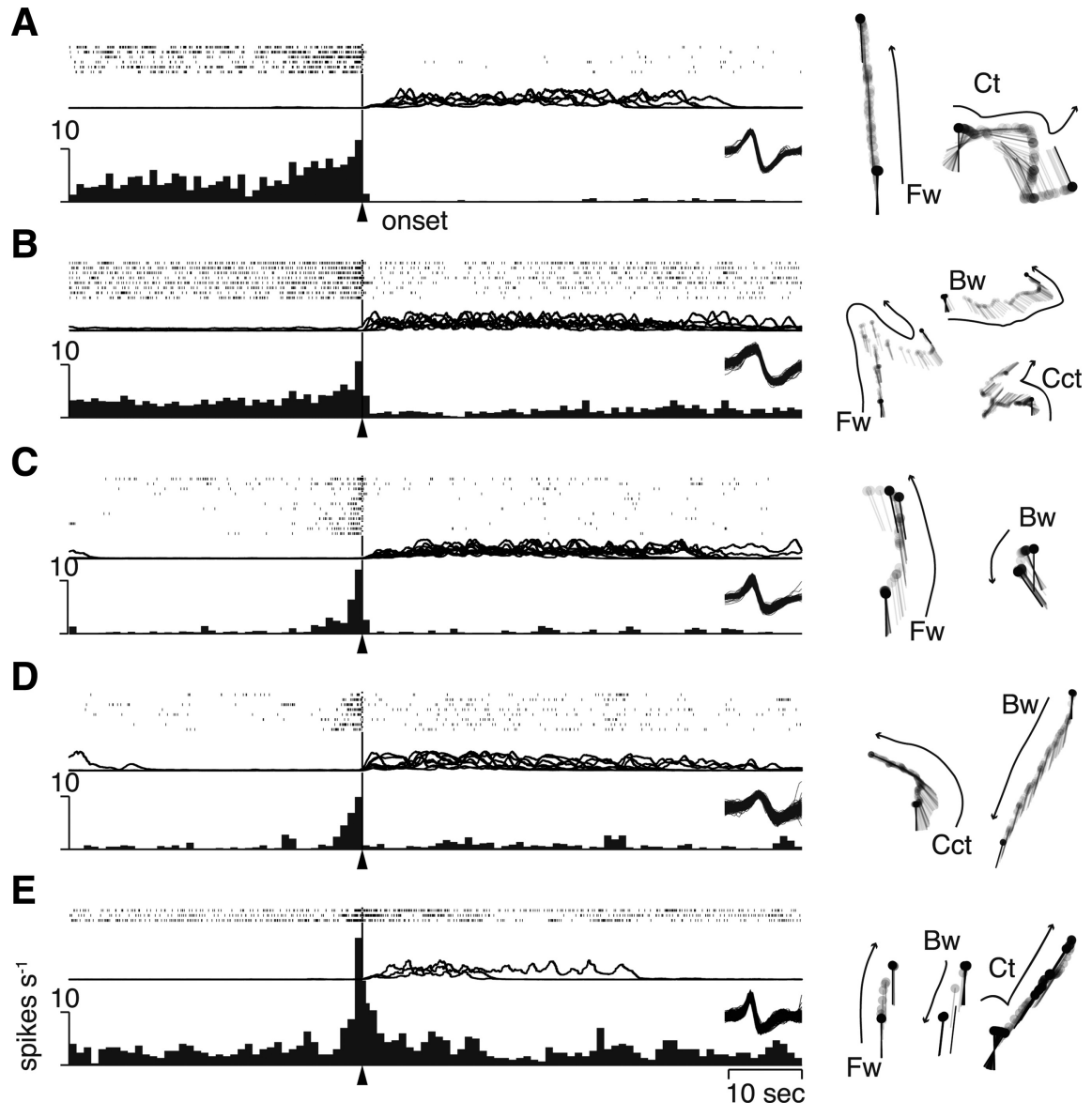

Figure 3. Readiness discharge activities from five different animals. $\boldsymbol{A}-\boldsymbol{E}$, Activity profiles of each readiness discharge unit. All units were obtained in the ventromedial quarter of the commissure. On the top of each panel are shown rastered spike trains, with the superimpositions of smoothed EMG below them. The perimovement time histogram is shown in the bottom. All the behavioral acts displayed by each animal during the recording session were categorized into four walking patterns and are shown on the right. Fw, Forward walking; Bw, backward walking; Ct, clockwise turning; Cct, counterclockwise turning.

locomotor behavior in general (Fig. 3). In other cases, however, we encountered those units whose spike activity showed a significant increase before a specific type of locomotor behavior. The descending unit illustrated in Figure $5 \mathrm{~A}$ showed an increase in the firing rate before the spontaneous initiation of forward walking. But it showed no noticeable change when the animal initiated backward walking. In contrast, another descending unit from another animal (Fig. 5B) showed an increased firing rate before the spontaneous initiation of backward walking. Note that, at the end of forward walking, this unit showed an increase in the spike discharge frequency followed by a bit of backward walking (marked by an asterisk in Fig. 5B). The directional selectivity of all 72 descending units from 10 animals is illustrated in Figure 5C. In the premovement period of $2 \mathrm{~s}$ just preceding the behavioral onset, 15 units including those indicated by black and gray arrowheads tended to show directional selectivity (Fig. 5C, left). After the animal initiated walking, however, almost all units including those indicated by arrowheads showed no direction-selective spike activity (Fig. $5 C$, right).

To clarify quantitatively how the selective and nonselective units are recruited temporally when the animal initiates and maintains walking behavior, we analyzed the number of each type of units before and after the onset time of walking. Fortyeight of all 72 units were found to show activity change before and/or after the behavioral onset of spontaneous walking. It was revealed (Fig. 5D) that the nonselective units began to be recruited $\sim 4$ s before the behavioral onset and then the selective units followed $\sim 2.5 \mathrm{~s}$ later. Both types of unit continued to increase gradually in number and reached a plateau $\sim 3 \mathrm{~s}$ after the behavioral onset in the middle of a walking bout. In the course of walking in either direction, not only the selective units for that direction but also the nonselective units were kept active, suggesting the critical role of nonselective units in maintaining the walking in either direction. The termination unit was found to increase its spike activity regardless of the stepping direction.

\section{Descending activities for spontaneous termination of walking}

We encountered a descending unit that increased its firing rate before the termination of walking in two animals. The recording shown in Figure $6 \mathrm{~A}$ was obtained when the animal first walked backward and then forward and finally stopped walking all spontaneously. This descending unit showed an increased firing rate before their termination regardless of the walking pattern in 6 of all 11 bouts of walking including straight forward, backward, and curved forward walking (Fig. $6 B$ ). It was interesting to note that the unit activity showed no noticeable change when the animal switched from backward to forward walking (Fig. $6 \mathrm{~A}$, horizontal line). This descending unit thus appeared to convey information on spontaneous termination of walking to the thoracic ganglia.

We confirmed that the unit showed no noticeable change in their spike activity before behavioral initiation of walking (Fig. $6 C$ ) just as the readiness discharge unit showed no activity change before behavioral termination of walking (Fig. 2D). Both the readiness discharge and termination units increased their spike activity before behavioral initiation and termination, respectively, and never did so in any other timing. No other unit than the readiness discharge and termination units increased its spike activity before the behavioral onset and offset. Additional analyses, in which arbitrary time points, instead of behavioral onset/ offset, were chosen for the alignment time of spike activity comparison, confirmed that no unit showed a noticeable increase in the spike discharge rate before the arbitrary time point demonstrating that the activity increase before behavioral onset/offset was not an artifact caused by chance.

\section{Behavioral effects of readiness discharges}

To test whether the spike activity of a readiness discharge unit is causally related to the initiation of walking behavior, we electrically stimulated a small bundle containing the unit and analyzed its behavioral effect using EMG and treadmill recordings. In the experiment illustrated in Figure 7, a small nerve bundle containing a putative readiness discharge unit was split off from the circumesophageal commissure on the right side. The commissure on the other side remained intact. An inset at the top right 


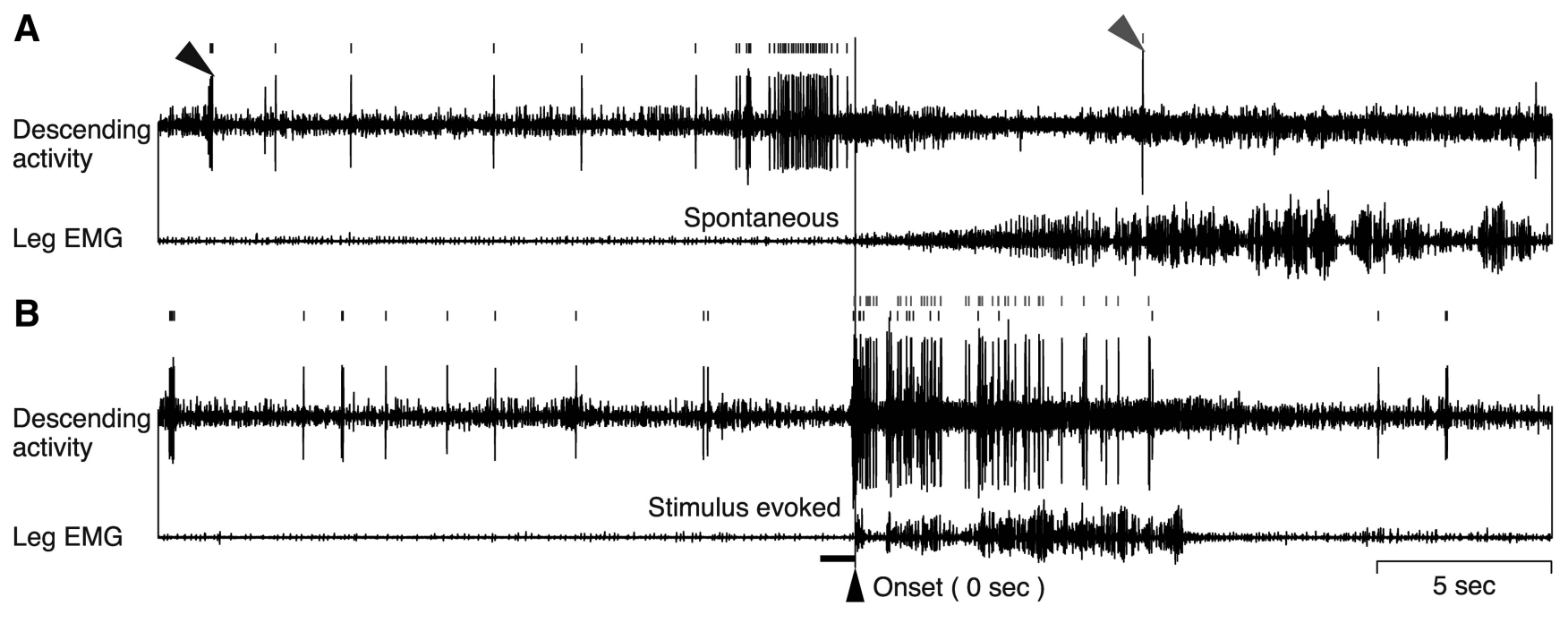

C

$-1.0 \sim 0 \mathrm{sec}$

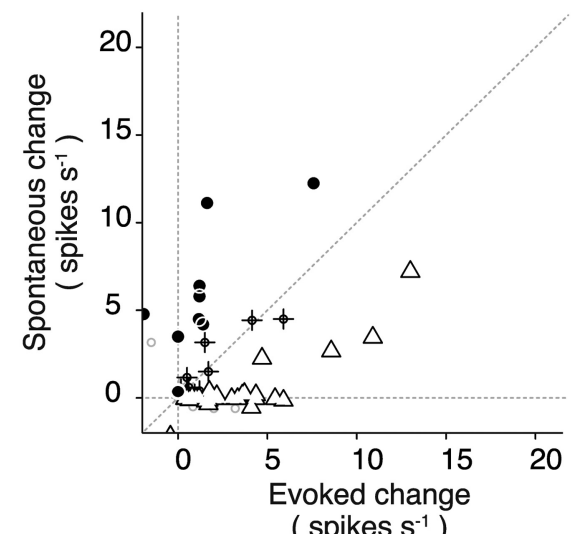

( spikes $\mathrm{s}^{-1}$ )

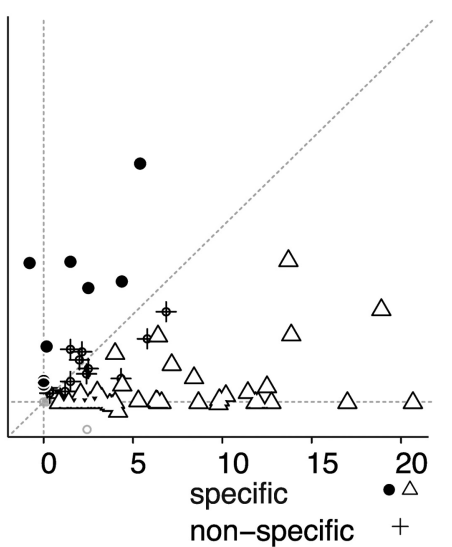

D

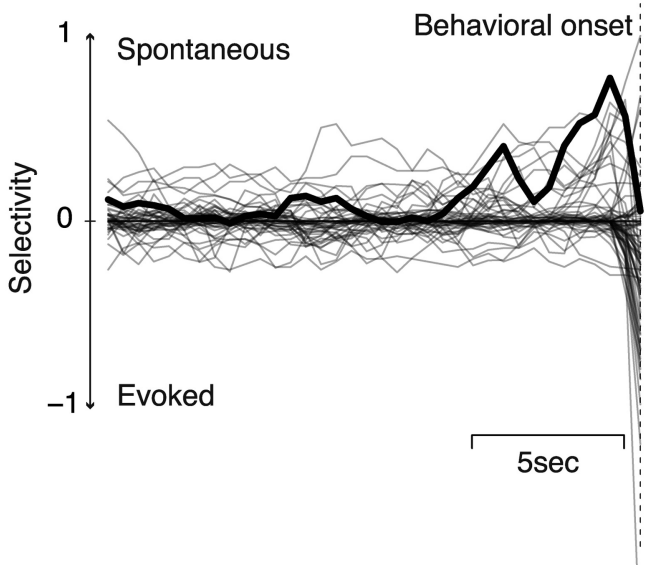

Figure 4. Selective activity of descending units for spontaneous and mechanical stimulus-evoked walking. $\boldsymbol{A}$, Descending unit activities recruited for the spontaneous initiation of walking. $\boldsymbol{B}$, Unit activities associated with mechanical stimulus-evoked initiation of walking recorded from the same animal as in $\boldsymbol{A}$. The horizontal bar on the left side of the behavioral onset indicates the approximate period of mechanical stimulation. Two different units were selectively recruited for the spontaneous (black arrowhead and spike trains) and stimulus-evoked (gray arrowhead and spike trains) walking. C, Activity changes in the descending units associated with spontaneous or mechanical stimulus-evoked initiation of walking. Each symbol represents the averaged difference in the spike discharge number of each unit between the control and the test period before the spontaneous and stimulus-evoked walking, plotted against the ordinate for the former and the abscissa for the latter type of walking. The control period was $1 \mathrm{~s}$ in duration, starting at 20 s before the behavioral onset, and common to the left and right panel of $\boldsymbol{C}$. The test period was $1 \mathrm{~s}$ in duration and started $1 \mathrm{~s}$ (left) or $0.5 \mathrm{~s}$ (right) before the behavioral onset. If the difference in the spontaneous or mechanical stimulus-evoked walking was judged to be statistically meaningful by model selection, the unit was classified into spontaneous (filled circle) or evoked walking-specific unit (open triangle), respectively. The unit that showed a meaningful difference in both types of walking behavior was plotted on or near the diagonal and classified into the nonspecific unit (cross). The test period for the right panel could detect larger number of evoked walking-specific units that increased spike activity just before or almost at the same time as the behavioral onset. $\boldsymbol{D}$, Temporal changes in the selective activity of descending units before the behavioral onset of spontaneous and mechanical stimulus-evoked walking. The test period was shifted from 19 to $0.5 \mathrm{~s}$ before the behavioral onset (from -19 to $-0.5 \mathrm{~s}$ ) with the time step of $0.5 \mathrm{~s}$. Each of the superimposed 81 traces shows the temporal change of behavioral selectivity of each unit obtained from 12 animals. We measured the distance from the diagonal to quantify the behavioral selectivity of unit discharges for the test period at each time. The ordinate is the measure of distance of each unit from the diagonal, normalized to the maximal value in the spontaneous domain to indicate the behavioral selectivity of the unit. The zero level means that the unit showed no selective activity for either spontaneous or evoked behavior. The trace deflects upward from zero when the unit increases its spike discharge at a certain time before the onset of spontaneous walking, whereas it deflects downward when the unit increases its spike activity at that time before the onset of mechanical stimulus-evoked walking. The abscissa indicates time. The measurement in each of the successive test period with time step of $0.5 \mathrm{~s}$ was connected with its neighbors by a straight line to make up the behavioral selectivity profile of the unit over the prebehavioral time period. The unit illustrated in $A$ is indicated by a thick line.

shows the location of the bundle on the standard map of Wiersma (1958). The bundle was then severed by a pair of microscissors to yield a rostral cut end from which descending unit activities were recorded extracellularly, and a caudal cut end to which a suction electrode was applied to stimulate the readiness discharge unit electrically. The experimental setup is schematically illustrated at the top left. The unit was relatively large in amplitude in the bundle recording (Fig. 7A, top trace). On its stimulation when the animal was at rest, a slow tonic increase was recorded fol- lowed by rhythmical bursts in the leg EMG activity (second trace). This activation pattern is characteristic to spontaneously initiated walking (Fig. 4A) (Chikamoto et al., 2008). The leg activity caused backward walking as recorded by the treadmill sphere movements (third trace). We could stimulate the unit 11 times in the same animal to confirm its consistent effect on the EMG activity (Fig. 7A, bottom trace) and on the animal behavior (Fig. $7 B$ ). The stimulated bundle was confirmed to contain a readiness discharge unit by its characteristic activity before the 


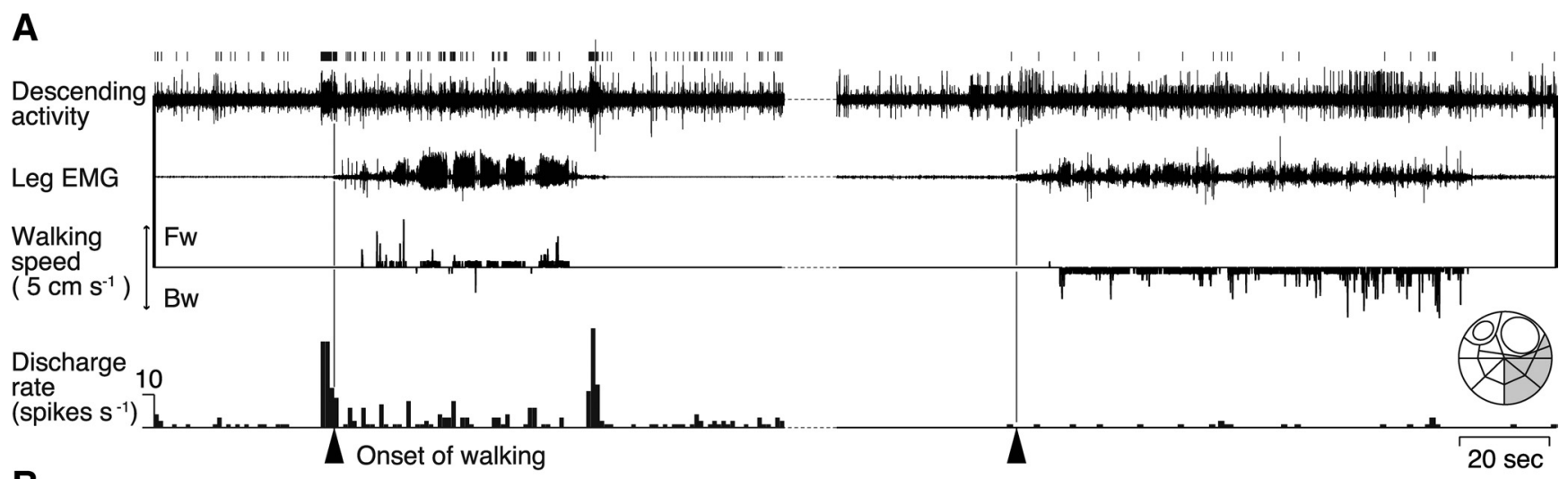

B

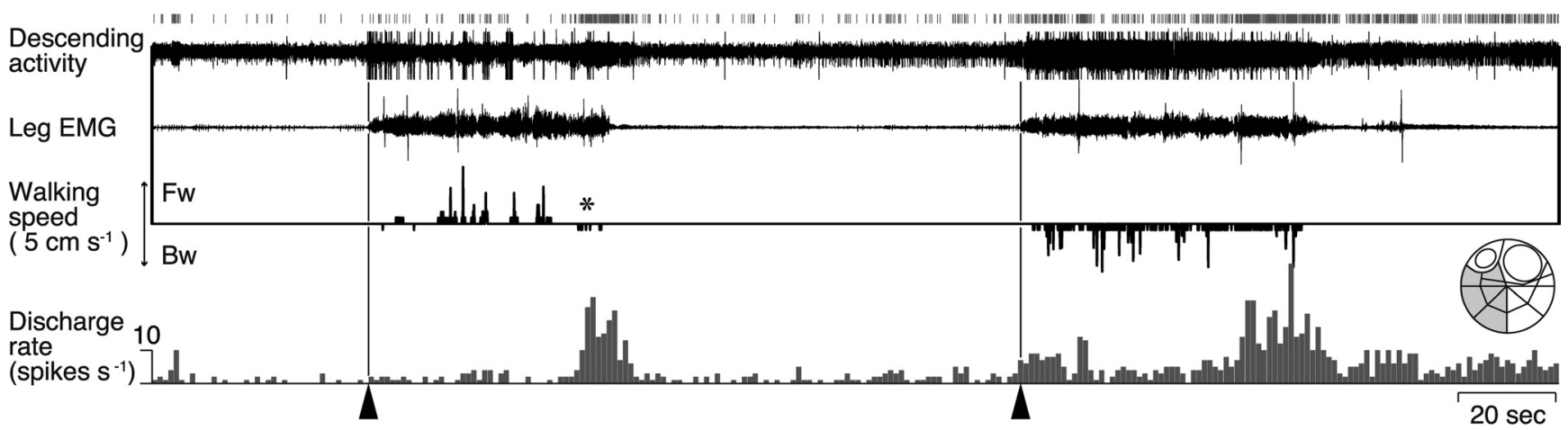

C
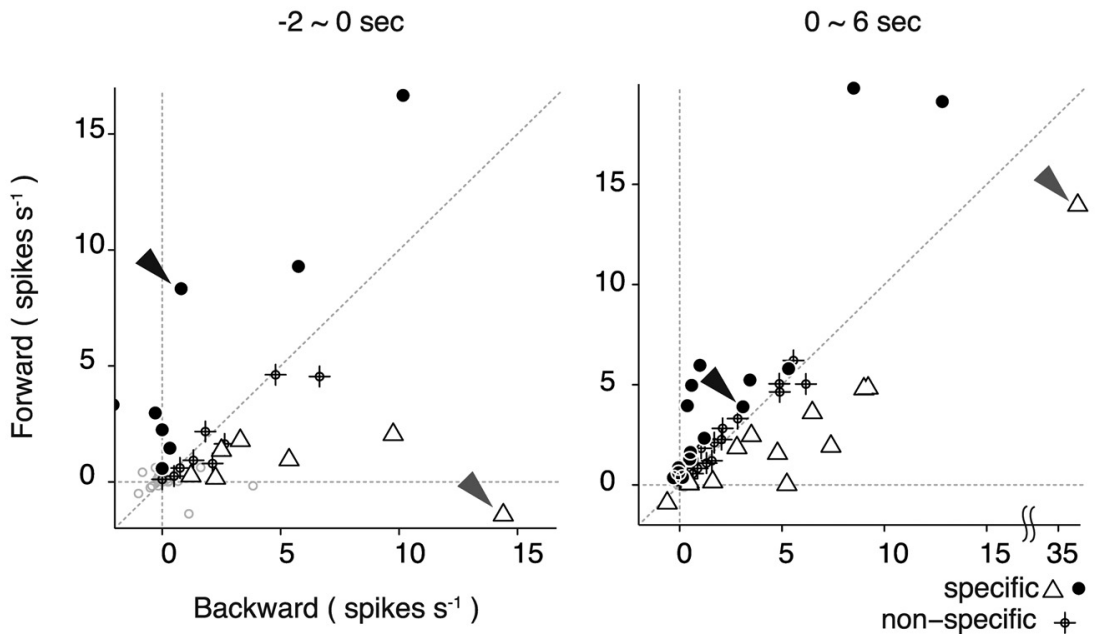

D

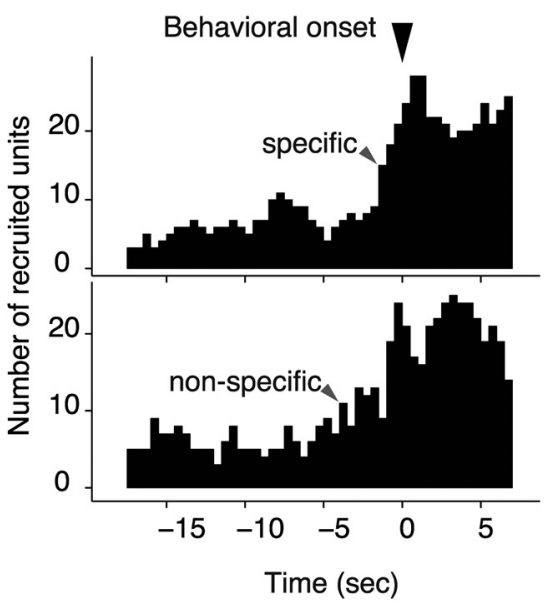

Figure 5. Selective activation of descending units for spontaneous initiation of forward and backward walking. $A$, Descending unit activities associated with forward walking. On the top are shown the spike trains together with the bundle recording, the EMG, and treadmill movements shown below. The perimovement time histogram of the unit activity is shown at the bottom. Recording was made from a small bundle isolated from the ventrolateral region of the circumesophageal commissure (inset on the far right). B, Descending unit activities associated with backward walking. C, Selectivity of 72 descending units for forward (ordinate) and backward walking (abscissa). Each symbol represents the averaged difference in the spike discharge number of each unit between the control and the test period before the spontaneous walking in the forward and backward direction, plotted against the ordinate for the former and the abscissa for the latter direction. The control and the test periods were 2 s in duration, starting at 20 and 2 s before the behavioral onset, respectively, in the left panel of $C$. To analyze the unit activity during walking, the control and the test periods were $6 \mathrm{~s}$ in duration, starting at $20 \mathrm{~s}$ before and just at the behavioral onset in the right panel. If the difference before the forward or backward walking was judged to be statistically meaningful by model selection, the unit was judged to be forward- (filled circle) or backward-selective (open triangle). The unit that showed differences before walking in either direction was judged to be directionally nonselective (cross). The unit indicated by a black arrowhead corresponds to the one shown in $\boldsymbol{A}$, whereas that indicated by a gray arrowhead to the one shown in $\boldsymbol{B}$. $\boldsymbol{D}$, Number of directional and nondirectional units recruited in association with self-initiated walking. The $2 \mathrm{~s}$ test period was shifted from $18 \mathrm{~s}$ before the behavioral onset $(-18)$ to $6 \mathrm{~s}$ after it $(+6)$ by $0.5 \mathrm{~s}$. Each unit was judged to be either direction-specific or nonspecific by model selection at every test period. The top histogram shows the number of directionally specific units, whereas the bottom histogram shows that of nonspecific units along time. The specific units began to be recruited at $1.5 \mathrm{~s}$ before the behavioral onset as indicated by an arrowhead, whereas the nonspecific units were recruited earlier, at 4.5 s before the onset.

initiation of walking behavior (Fig. 7C). The spike trains recorded in five bouts of spontaneous walking (Fig. 7C, top trace) whose EMG recordings are all superimposed (middle trace) were used to compile the spike activity histogram (bottom trace) that dem- onstrates the readiness discharge preceding the behavioral onset of walking.

Since the electrical stimuli were always applied in the present study to a small bundle containing the relevant functional unit 


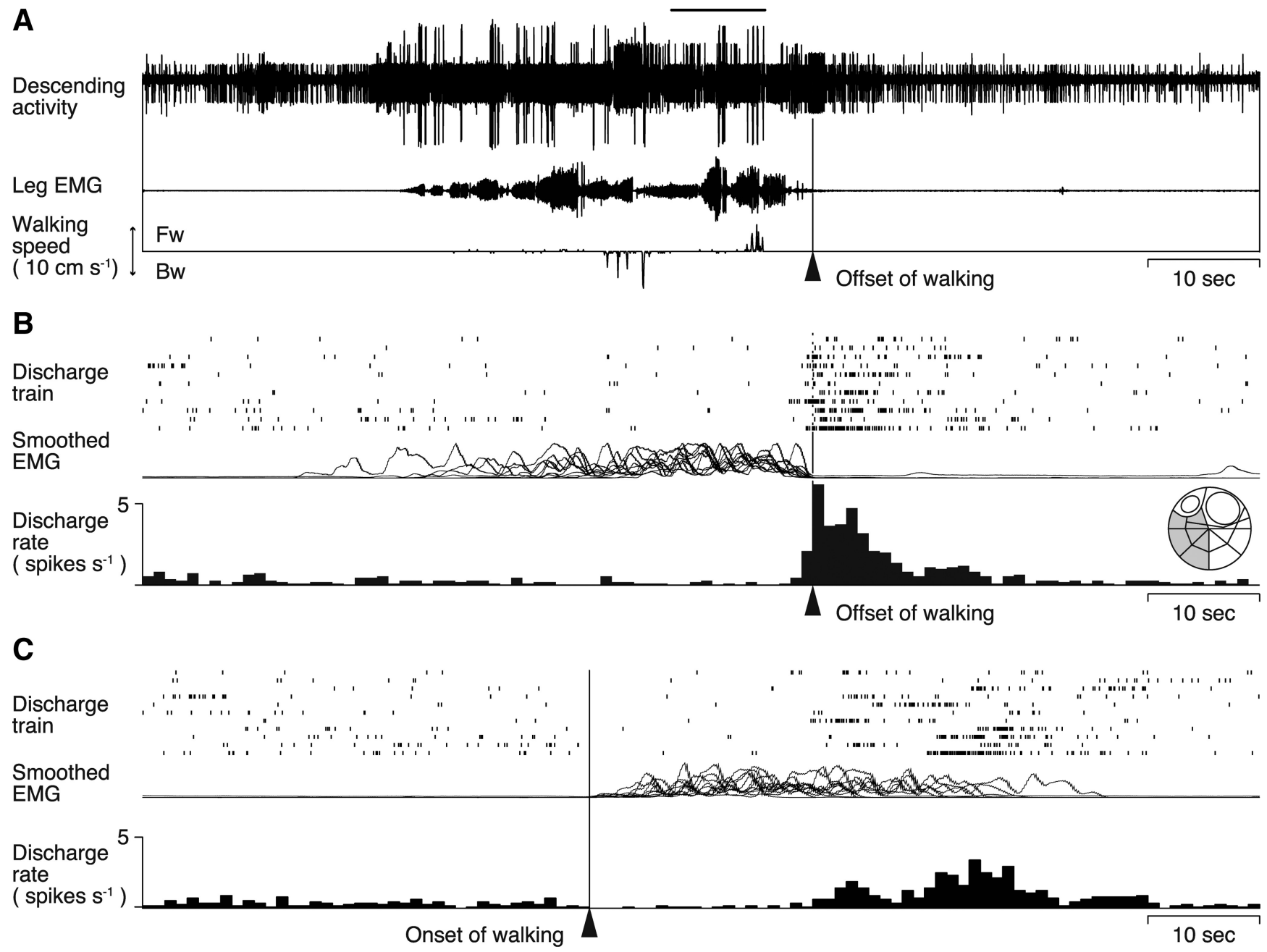

Figure 6. Descending unit activities before spontaneous termination of walking. $A$, On the top is the bundle recording with the EMG and treadmill movements shown below. The offset time of walking is shown with an arrowhead. $\boldsymbol{B}$, Activity profiles of a unit isolated from the bundle recording. The rastered spike trains are shown with the superimposition of integrated EMGs and a perioffset time histogram of the unit activity. The inset on the far right shows the location of the recorded bundle on the cross- sectional map of Wiersma (1958) of the circumesophageal commissure. The recording shown in $A$ was obtained when the animal first walked backward and then forward and, finally, stopped walking all spontaneously. This descending unit showed an increased firing rate in 6 of all 11 bouts of walking before its termination regardless of the walking pattern, including straight forward, backward, and curved forward walkings. C, Unit activities aligned to the onset time of walking. No activity change is observed before the behavioral onset.

instead of the unit itself, there remained the possibility that the stimulation affected other neurons than the targeted one. The stimulated bundle in the experiment illustrated in Figure 7, for instance, contained not only a large readiness discharge unit but also two other small units that showed an increase in their spike activity when the animal initiated walking behavior, and maintained the increased activity as long as the animal kept walking (Fig. $7 A$, top trace). It was unlikely, however, that these small units were directly activated to cause walking since the electrical stimulation, which was limited to several seconds in duration, evoked the walking behavior and the small unit activities that lasted for $>60 \mathrm{~s}$ (Fig. 7A). Thus, the electrical stimulation activated the readiness discharge unit whose transient increase in the spike discharge rate evoked long-lasting walking behavior by recruiting tonic units, such as those observed in Figure $7 A$, that represented direction- and behavior-specific information for initiating and maintaining the walking behavior.

We could also test the behavioral output of one descending unit that was activated in association with the mechanical stimulus-evoked walking, and another unit that was activated before spontaneous termination of walking behavior in different animals. Electrical stimulation of the descending unit whose activity was associated with reflexively initiated walking consistently caused an increase in the muscle activity that was characteristic to this type of walking (Fig. 4B) (i.e., sudden commencement of rhythmical burst activities without any preceding slow tonic increase of the muscle activity) (Fig. 8). Electrical stimulation of the termination unit in another animal caused termination of ongoing walking (Fig. 9). These findings suggested that the descending units found in this study possess functional ability to cause behavioral changes by their spike activities.

The number of descending units recruited in association with spontaneous walking can be summarized as follows (Table 1). The readiness discharge, continuation, and termination units were judged on their spike activity during the period that started $2 \mathrm{~s}$ before and ended at the behavioral onset, that started at the behavioral onset and ended $6 \mathrm{~s}$ after it, and that started $1 \mathrm{~s}$ before and ended at the behavioral offset, respectively. The units were classified into direction-selective and nonselective groups and the former further into forward- and backward-selective groups. After 72 units from 10 animals were tested against these categories, 48 units were found to increase their spike activity when the 


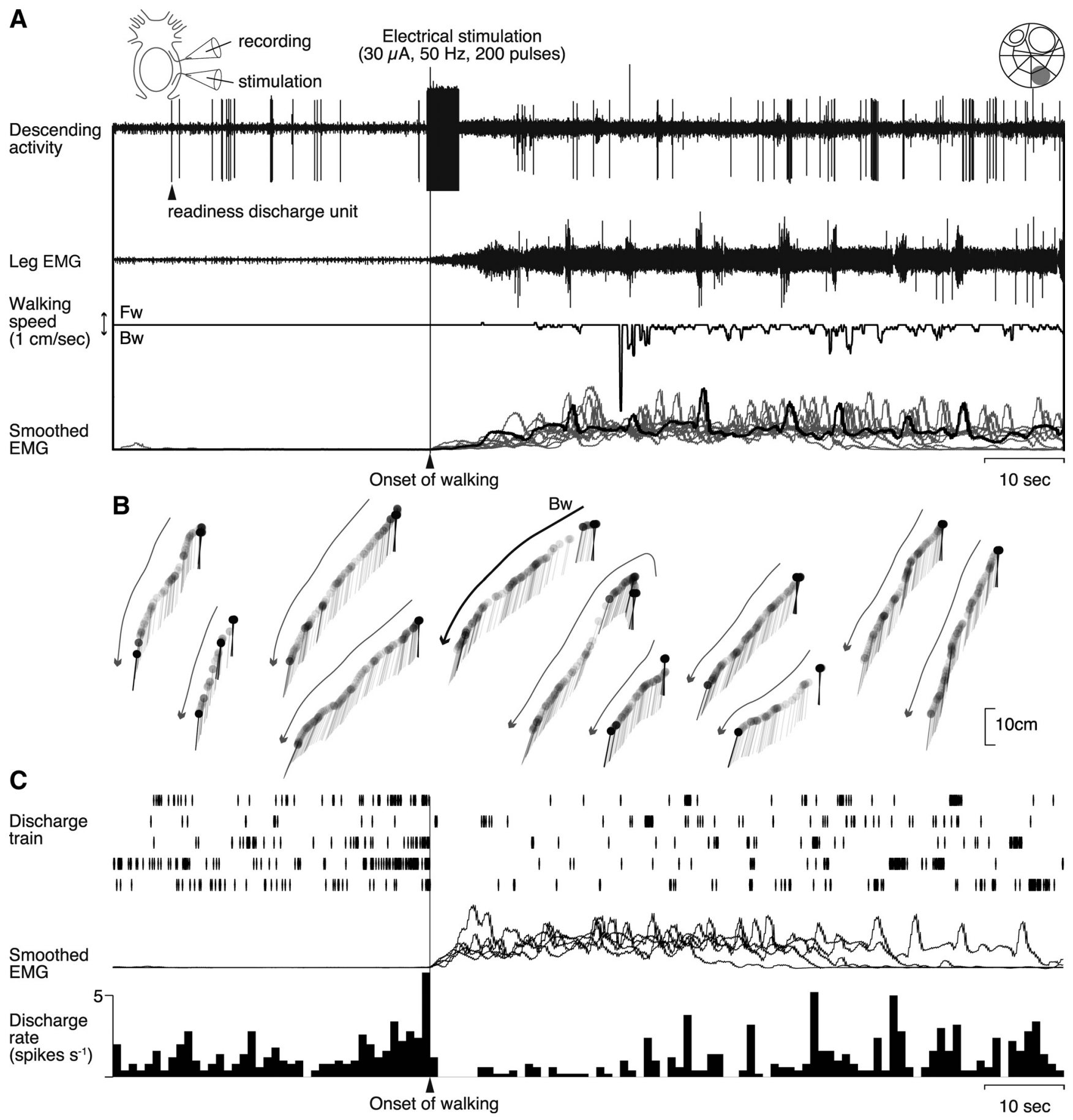

Figure 7. Walking behavior initiated by electrical stimulation of a readiness discharge unit. $A$, Effects of electrical stimulation on the leg muscle activity. The stimulus artifact in the rostral cut end recording (top trace) indicates the onset and offset time of the stimulus ( 200 pulses of $0.2 \mathrm{~ms}$ duration at $50 \mathrm{~Hz}, 30 \mu \mathrm{A}$ ). The stimulation first caused a slow tonic increase followed by rhythmical bursts in the EMG activity recorded from the mero-carpopodite muscle of the second right leg (second trace). Leg movements resulted from the muscle activity were recorded as the movements of the treadmill sphere (third trace). Downward deflection indicates backward walking. At the bottom are superimposed all $11 \mathrm{EMGs}$ (gray and black traces) that were integrated electronically with the time constant of $1 \mathrm{~s}$, including the one corresponding to the record shown in the second trace (black line). All records were obtained from the stimulation of the same bundle. $\boldsymbol{B}$, Displacement of the animal body reconstructed from the treadmill sphere movements. The curved arrows indicate the walking direction in each stimulation. The bold one corresponds to the experiment shown in $\boldsymbol{A}$. Abbreviations are the same as in Figure 1. C, Activity profiles of the readiness discharge unit. Before the stimulation experiment, the descending spike activity of the unit was confirmed in the rostral cut end recording to precede the behavioral onset of spontaneously initiated walking. At the top are shown five traces of the spike trains. The integrated EMG activities are superimposed in the middle. The spike activity preceding the behavioral onset of walking is illustrated in the histogram at the bottom compiled from the spike trains shown above. All data in $A-C$ were obtained from the same animal.

animal initiated walking spontaneously, whereas 24 other units never showed an increased activity associated with the behavior. Eight units showed an increase in the spike discharge rate just before the animal spontaneously initiated forward walking and seven units before backward walking. Nine other units increased spike activities before the animal initiated walking in either direction. Sixteen units, including 6 units whose activity increase lead the behavioral onset time, maintained spike activities when the 


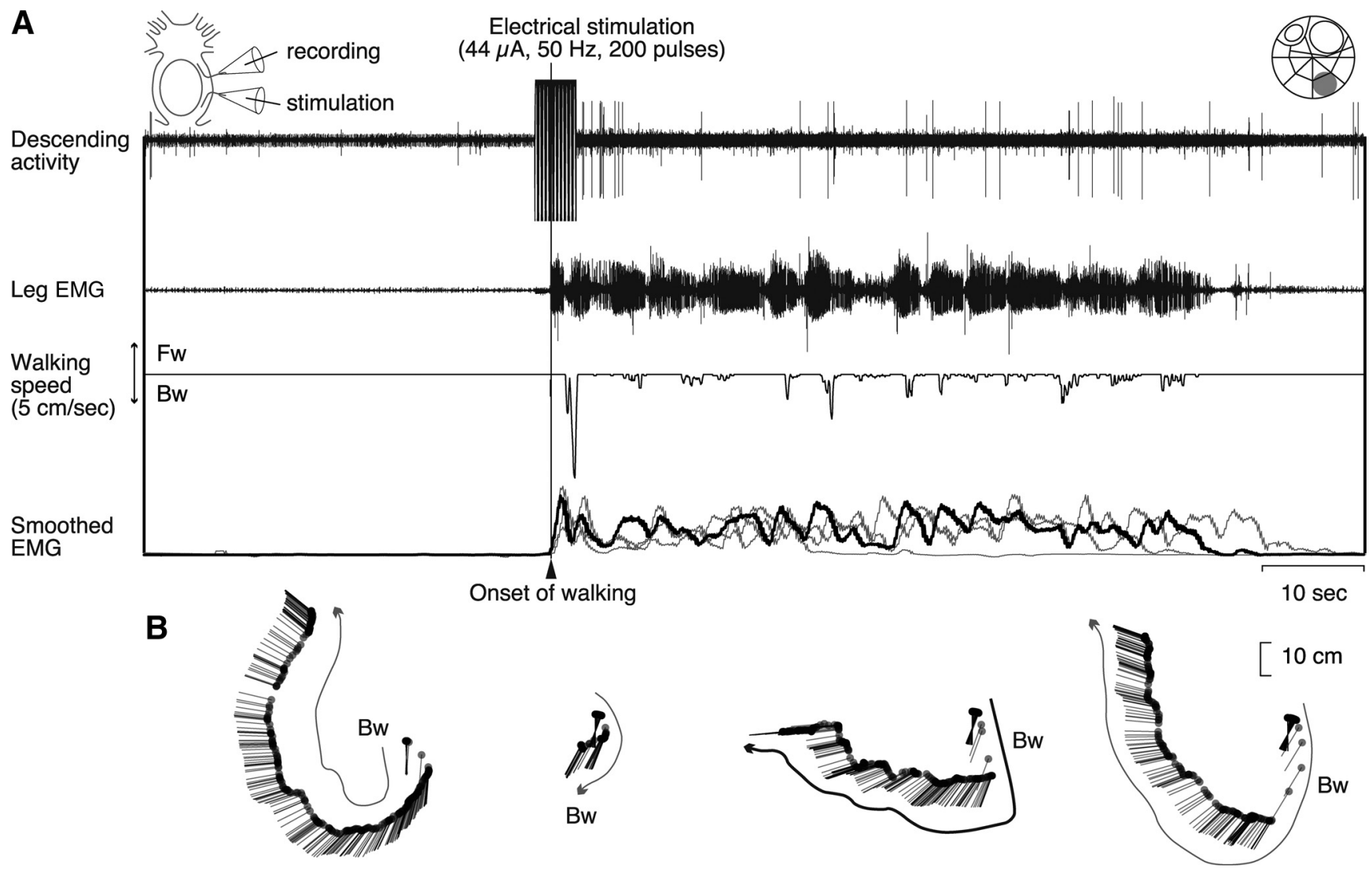

Figure 8. Electrical stimulation of a descending unit associated with reflexively evoked walking. We confirmed that the animal showed walking behavior when a small bundle (shown at the top right), which contained a descending unit activated in the stimulus evoked walking, was stimulated electrically. This walking was similar to that observed in the natural walking, which was evoked reflexively by mechanical stimulation of the telson, but was different from that observed in the spontaneous walking. The experimental setup is schematically illustrated at the top left. $A$, Effects of electrical stimulation on the leg muscle activity and animal behaviors. The stimulus artifact in the rostral cut end recording (top trace) indicates the onset and offset time of the stimulus (200 pulses of $0.2 \mathrm{~ms}$ duration at $50 \mathrm{~Hz}, 44 \mu \mathrm{A}$ ). The stimulation first caused a fast phasic increase followed by rhythmical bursts in the EMG activity recorded from the mero-carpopodite muscle of the second right leg (second trace). Leg movements resulted from the muscle activity were recorded as the movements of the treadmill sphere (third trace). Downward deflection indicates backward walking. At the bottom are superimposed all four EMGs (gray and black traces) that were integrated electronically with the time constant of $1 \mathrm{~s}$, including the one corresponding to the raw record shown in the second trace (black trace). All records were obtained by stimulation of the same bundle. $\boldsymbol{B}$, Movements of the animal body reconstructed from the treadmill sphere movements. The curved arrows indicate the walking direction in each stimulation. The bold one corresponds to the experiment shown in $A$. Abbreviations are the same as in Figure 1.

animal walked in the forward direction and 13 units including 7 leading units in the backward direction. Another 18 units including 10 leading units showed sustained spike activities during walking in either direction. One unit increased its spike activity before the offset of walking in either direction. In addition to those units summarized above, there were other units obtained in animals that showed only a fraction of the whole behavioral repertoire (e.g., only forward or backward walking or turning). They were classified into the provisional group in this study and the distinction of directional selectivity or nonselectivity could not be made for these units. Thirty-four provisional units increased their spike discharge rate before the onset of either forward or backward walking or before turning behavior in any direction, and 54 provisional units including 33 units that increased spike discharges before the behavioral onset maintained spike discharges when the animal kept walking or turning. Two provisional units increased their spike discharges before the offset of forward walking. The functional roles of those descending pathways including the readiness discharge units are hypothetically summarized in Figure 10, which illustrates the current conceptual framework regarding the neural control of spontaneously initiated locomotor behavior in crayfish.

\section{Discussion}

Our results demonstrated that neuronal activities generated internally precede the behavioral onset of self-initiated walking in crayfish. Those activities, termed readiness discharges, were related to no specific direction of walking, representing some internal processes within the brain that were generically related to the spontaneous initiation of locomotor behavior (Fig. 3). In addition to the readiness discharge units, we also found those descending cells that were selectively activated during forward or backward walking and those that showed activity increases before the termination of walking (Figs. 5, 6). Furthermore, we confirmed the causal effect of readiness discharge and termination units on the crayfish behavior by electrical stimulation experiments (Figs. 7-9). The readiness discharge units were never observed to be activated before reflexive initiation of walking in response to mechanical stimulation. Instead, other descending units were recruited in association with mechanical stimulusevoked walking (Fig. 4). It remains unknown, however, whether the chemical stimulus-evoked walking, which showed similar EMG activation pattern to that of spontaneously initiated walking (Chikamoto et al., 2008), is mediated by the readiness discharge units or by other separate ones. In any case, our findings 


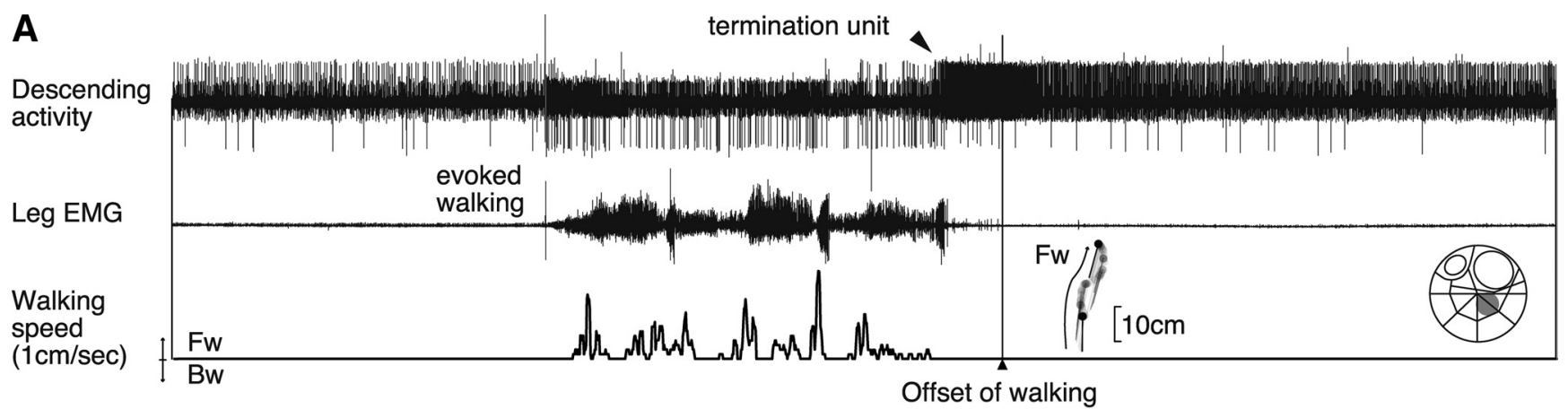

\section{B}

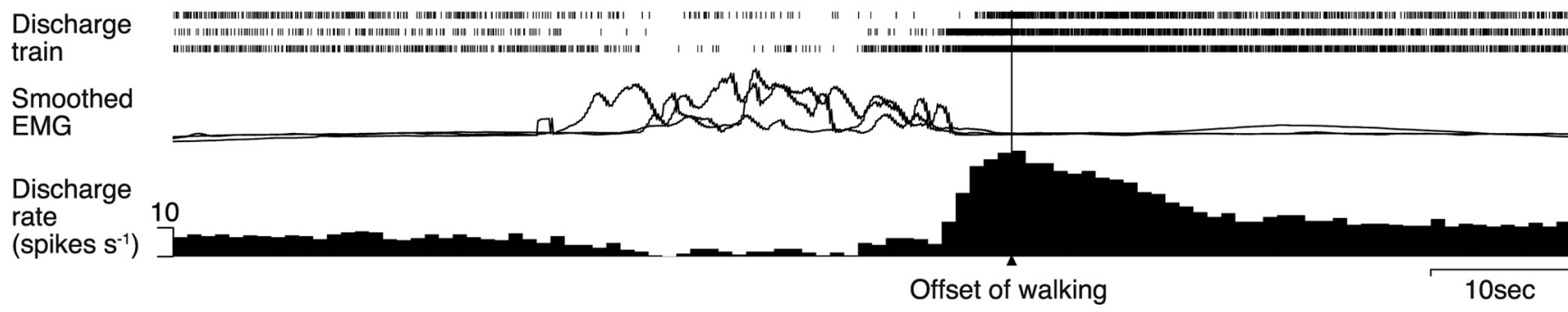

C $31 \mu \mathrm{A}, 50 \mathrm{~Hz}, 200$ pulses

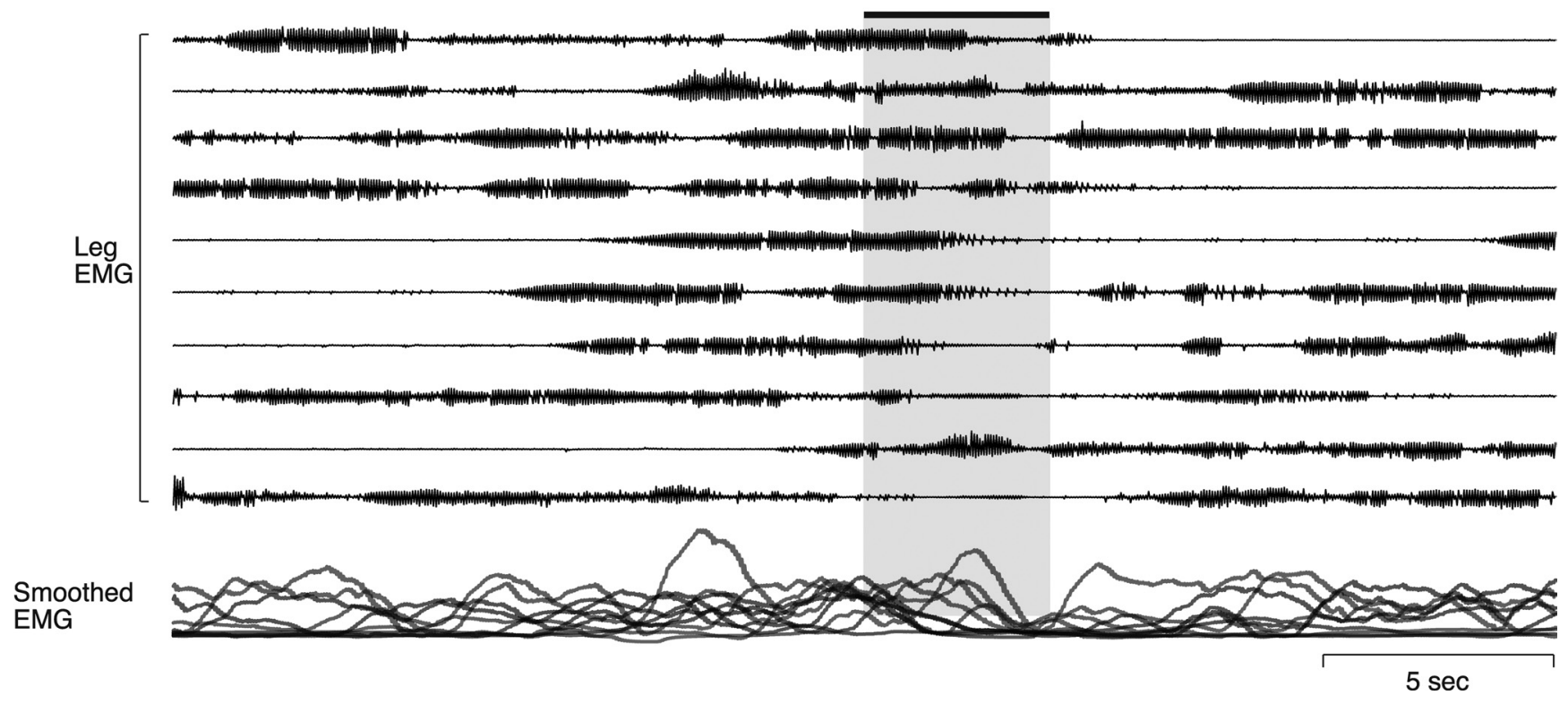

Figure 9. Electrical stimulation of a descending unit associated with spontaneous termination of walking behavior. A, Spike activity of the descending unit recorded when the animal initiated walking in response to mechanical stimulation of the telson and terminated spontaneously. The unit activity (top trace, arrowhead) increased before the decrease of leg muscle activity (second trace). $\boldsymbol{B}$, Activity profiles of the termination unit compiled from three bouts of walking. $\boldsymbol{C}$, Behavioral effects is caused the electrical stimulation of the bundle containing the termination unit during walking. Electrical stimulation ( 200 pulses of $0.2 \mathrm{~ms}$ duration at $50 \mathrm{~Hz}, 31 \mu$ A shown in solid horizontal bar at top) was applied to the bundle at 10 different timings during walking behavior. At the bottom are superimposed smoothed EMGs, indicating a decrease in the leg muscle activity after the electrical stimulation (shown by the gray area).

suggest that a parallel descending system consisting of these functional units would causally contribute, at least partly, to spontaneous initiation, continuation, and termination, as well as reflexive initiation, of walking. This kind of parallel organization of descending units for initiating and controlling locomotor behavior is comparable with that proposed in the vertebrate nervous system (Grillner et al., 2008; Jordan et al., 2008). In the following sections, we discuss the hierarchical and modular features in the parallel descending system for locomotor behavior of crayfish.
Behavioral hierarchy for the self-initiated walking

A number of studies have been conducted to demonstrate the existence of command neurons or systems for a variety of behavioral acts in invertebrates (Clarac and Pearlstein, 2007; Kristan and Gillette, 2007) by testing whether a nerve cell or a set of them can elicit a specific behavior on stimulation and whether they are active or not during behavior as well as whether they are activated by sensory stimuli or not. However, there are few studies on how the command neurons are activated when an animal initiates a specific behavior spontaneously. In this study, we found for the 
Table 1. Number of functional units associated with spontaneous walking

\begin{tabular}{llcll}
\hline Directionality & Direction & Initiation & Continuation & Termination \\
\hline Selective & Forward & $8(6)$ & $16^{a}(8)$ & - \\
& Backward & $7(6)$ & $13^{b}(7)$ & - \\
Nonselective & & $9(5)$ & $18^{c}(8)$ & $1(1)$ \\
Provisional & & $34(12)$ & $54(13)$ & $2(2)$ \\
\hline
\end{tabular}

Those units obtained in animals that showed only a fraction of the whole behavioral repertoire (e.g., only forward or backward walking) were classified as provisional since the distinction of directional selectivity and nonselectivity could not be made for these units. The number of animals for each type of units is shown in parentheses. ${ }^{a}$ Including 6 units that increased spike activity prior to initiation of walking in the forward direction. ${ }^{b}$ Including 6 units that increased spike activity prior to initiation of walking in the backward direction. Including 8 units that increased spike activity prior to initiation of walking in either direction.

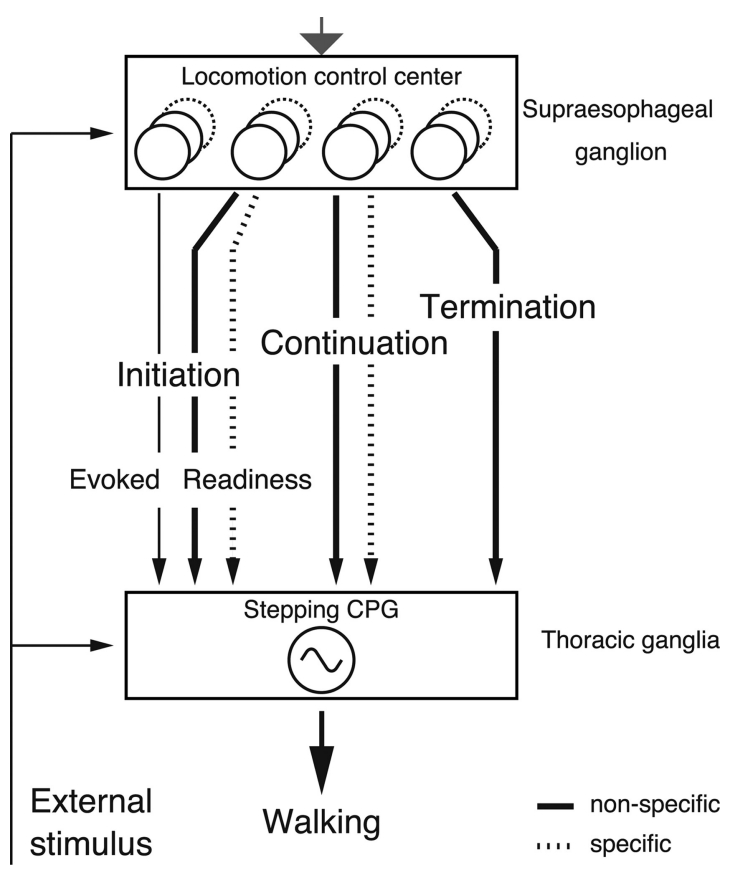

Figure 10. Schematic drawing of the current conceptual framework on the functional roles of descending units in the control of walking behavior in crayfish. Descending pathways for spontaneous initiation of locomotor behavior consist of directionally nonspecific (thick solid line) ones for readiness discharge units and specific ones for directionally specific (dotted line) ones for continuation units. The nonspecific and specific pathways are organized in parallel, and the former is recruited earlier than the latter. The pathway for mechanical stimulus-evoked walking (thin solid line) was distinct from those for spontaneously initiated walking. During walking, other units were active in a sustained way, either directionally nonspecific (thick solid line) or specific (dotted line). Some of them are responsible for maintaining walking, whereas others reflect, at least partly, corollary discharges associated with the locomotor activity. Before the termination of walking, a distinct group of descending units is recruited. The locomotor behavior of crayfish is thus controlled by descending units organized in parallel structurally and in hierarchy functionally. The gray arrow on the top indicates hypothetical input from motivational centers for feeding, mating, exploration, etc.

first time in crayfish, in which a variety of command fibers have been described to initiate behavior on stimulation (Bowerman and Larimer, 1974a,b), the preparatory or readiness spiking activity in the descending pathway from the brain to thoracic ganglia where stepping generators are located. Those activities preceded the behavioral onset by $>1 \mathrm{~s}$. Neural recordings of other spontaneous activity may show a shorter latency (Garcia-Perez et al., 2007) because they represent motoneuronal rather than interneuronal activity. For understanding the functional significance of readiness discharges in crayfish behavior, the scheme of behavioral hierarchy will be useful, in which an animal's decision to act proceeds from a high level for general behavior to lower levels for special actions (Tinbergen, 1951; Davis, 1979).
The readiness discharge was found to be a good predictor of spontaneous initiation of walking, but it was not related to the stepping direction (Fig. 3), thus representing a general decision to start the locomotor behavior. This activity can be comparable with those activities recorded in the primate striatum or supplemental motor area during internally generated preparation for arm movements (Schultz and Romo, 1992; Schultz et al., 1997). We also found those units that showed selective activities for either forward or backward walking as long as the animal maintained the behavior. Those activities were interpreted in this study to represent more specific decisions, occupying a lower level in the hierarchical organization of the decision making system for locomotor behavior. Directionally selective units began to be recruited before the behavioral onset, but later than the time when directionally nonselective units began to be recruited (Fig. $5 D$ ). It is suggested that the spontaneous initiation of locomotor behavior proceeds from a general decision to walk to more specific decisions on the walking direction.

Do the directionally selective units represent the command for walking in a specific direction? The question remains open, but a possibility is that they represent the final specific decision as the command neuron for walking in a specific direction. The bundle that elicited backward walking on electrical stimulation could contain a command fiber reported to be located in Wiersma's area 70 (Atwood and Wiersma, 1967). Alternatively, they might supervise the command neuron or system at a level higher than the specific command neurons but lower than the readiness discharges representing more general decisions on locomotor behavior. In any case, our finding suggest that the command neurons or systems that have been thought to be the highest motor element in the sensory-evoked behavior (Edwards et al., 1999) are ranked lower in the hierarchical organization of the decision making system when an animal initiates behavior spontaneously.

\section{Modular representations for sequential control of locomotion}

In general, the animal body is highly redundant in the degree of freedom of joint movements in such a way that, if animals are to control their body by themselves in a goal-directed way, they must solve hard computational problems regarding the trajectory and temporal profile of movement (Kawato, 1999; Bizzi et al., 2000). As a solution to this problem, animals have implemented modular organization in different levels of the motor control hierarchy (Flash and Hochner, 2005). Much evidence from motor systems of vertebrates and invertebrates supports the idea that the modular primitives reduce the dimensionality to simplify the complexities resulting from the redundancy (Bizzi et al., 2000; Flash and Hochner, 2005). However, it remains unknown how those modules are internally represented in the invertebrate CNS, especially when they initiate behavior spontaneously.

The readiness discharges that preceded spontaneous initiation of walking did not relate to any specific stepping direction in many cases (Figs. 3, 5D). They appear to be modularized at a general higher-order level for the macro-level control. Such a macro-level representation is in line with the decision-making system in the leech locomotion (Esch et al., 2002) and the Aplysia feeding (Jing et al., 2004), in which higher modules for initiation are activated by external stimuli to drive lower modules for pattern generation. Unlike them, however, higher modules for initiation of locomotor behavior in crayfish are driven not exogenously but endogenously. Although the higher module R3b1 of leech is also driven endogenously, it is different from the crayfish module in that it can be driven externally: the crayfish module 
can be driven only spontaneously (Fig. 4). This suggests that there exists some internal models in the crayfish brain regarding the spontaneous initiation of walking. Directionally nonselective readiness discharge units thus represent the general (i.e., not yet specifically determined) stage of neural activities in the brain associated with spontaneous initiation of walking. As for more specific aspects of the behavior, such as the direction and frequency of stepping movements, we could find those units that selectively increased their spike discharge rate during forward or backward walking, recruited later than the nonselective units (Fig. 5D). Those delayed units that are directionally selective and active throughout the walking behavior represent the executive stage of the internal process subserving the spontaneous initiation of walking in crayfish. Additional analysis is needed, however, to settle the problem of how the direction and other specific aspects of walking are represented in the spike activity of specific units descending from the brain to thoracic ganglia.

Whether the relatively simple invertebrate CNS has the internal model of self-initiated action has been discussed in the context of efference copy or corollary discharge (Webb, 2004). The continuation unit activity corresponds, at least partly, to the higher-order corollary discharge (Crapse and Sommer, 2008). There are two possibilities for the functional significance of this activity. First, it can be used for activating lower modules including central pattern generators (CPGs) that generate patterned rhythmical motor outputs; it has been shown that a sustained input is required for generation of rhythmic locomotor outputs (Grillner et al., 2008). Alternatively, it can be used for sensory processing to suppress reafferent signals and/or for potentiating other behavioral acts including postural reflexes that are subsidiary to walking (Hama et al., 2007). In any case, the unit activities for initiation, continuation, and termination of walking are temporally modularized and can be used to sequentially control the self-initiated walking behavior (Fig. 10). An animal can control the CPG activity at its own disposal by using these modules. It is interesting to note here that, in Xenopus tadpoles, the sensory evoked initiation and termination of locomotor behavior are mediated by distinct groups of descending units in the spinal code (Perrins et al., 2002). However, it is unknown how the modularized activities themselves are controlled by a higher center in the brain, if any. Additional study of brain neurons and their synaptic integration regarding those descending activities will provide deeper understanding of the spontaneous motor control mechanism as a potential manifestation of volition in invertebrates.

\section{References}

Akaike H (1974) A new look at statistical model identification. IEEE Trans Automat Contr 19:716-723.

Atwood HL, Wiersma CAG (1967) Command interneurons in the crayfish central nervous system. J Exp Biol 46:249-261.

Bizzi E, Tresch MC, Saltiel P, d'Avella A (2000) New perspectives on spinal motor systems. Nat Rev Neurosci 1:101-108.

Bowerman RF, Larimer JL (1974a) Command fibers in the circumesophageal connectives of crayfish: I. Tonic fibres. J Exp Biol 60:95-117.

Bowerman RF, Larimer JL (1974b) Command fibers in the circumesophageal connectives of crayfish: II. Phasic fibres. J Exp Biol 60:119-134.

Brodfuehrer PD, Thorogood MSE (2001) Identified neurons and leech swimming behavior. Prog Neurobiol 63:371-381.

Burrows M (1996) The neurobiology of an insect brain. Oxford: Oxford UP.

Chikamoto K, Kagaya K, Takahata M (2008) Electromyographic characterization of walking behavior initiated spontaneously in crayfish. Zoolog Sci 25:783-792.
Chrachri A, Clarac F (1990) Fictive locomotion in the fourth thoracic ganglion of the crayfish, Procambarus clarkii. J Neurosci 10:707-719.

Clarac F (2008) Some historical reflections on the neural control of locomotion. Brain Res Rev 57:13-21.

Clarac F, Pearlstein E (2007) Invertebrate preparations and their contribution to neurobiology in the second half of the 20th century. Brain Res Rev 54:113-161.

Crapse TB, Sommer MA (2008) Corollary discharge across the animal kingdom. Nat Rev Neurosci 9:587-600.

Davis WJ (1979) Behavioural hierarchies. Trends Neurosci 2:5-7.

Edwards DH, Heitler WJ, Krasne FB (1999) Fifty years of a common neuron: the neurobiology of escape behavior in the crayfish. Trends Neurosci 22:153-161.

Esch T, Mesce KA, Kristan WB (2002) Evidence for sequential decision making in the medicinal leech. J Neurosci 22:11045-11054.

Flash T, Hochner B (2005) Motor primitives in vertebrates and invertebrates. Curr Opin Neurobiol 15:660-666.

Garcia-Perez E, Mazzoni A, Torre V (2007) Spontaneous electrical activity and behavior in the leech Hirudo medicinalis. Front Integr Neurosci 1:8.

Gomes G (1999) Volition and the readiness potential. J Conscious Stud 6:59-76.

Grillner S, Wallén P, Saitoh K, Kozlov A, Robertson B (2008) Neural bases of goal-directed locomotion in vertebrates-an overview. Brain Res Rev 57:2-12.

Hama N, Tsuchida Y, Takahata M (2007) Behavioral context-dependent modulation of descending statocyst pathways during free walking, as revealed by optical telemetry in crayfish. J Exp Biol 210:2199-2211.

Jing J, Cropper EC, Hurwitz I, Weiss KR (2004) The construction of movement with behavior-specific and behavior-independent modules. J Neurosci 24:6315-6325.

Jordan LM, Liu J, Hedlund PB, Akay T, Pearson KG (2008) Descending command systems for the initiation of locomotion in mammals. Brain Res Rev 57:183-191.

Kawato M (1999) Internal models for motor control and trajectory planning. Curr Opin Neurobiol 9:718-727.

Kitagawa G, Takanami T, Matsumoto N (2001) Signal extraction problems in seismology. Int Stat Rev 69:129-152.

Kornhuber HH, Deecke L (1965) Hirnpotentialänderungen bei Willkürbewegungen und Passiven Bewegungen des Menschen: Bereitschaftspotential und Reafferente Potentiale. Pflugers Arch Gesamte Physiol Menschen Tiere 284:1-17.

Kristan W, Gillette R (2007) Behavioral choice. In: Invertebrate neurobiology (North G, Greenspan RJ, eds), pp 533-553. Cold Spring Harbor, NY: Cold Spring Harbor Lab.

Maimon G, Assad JA (2006) Parietal area 5 and the initiation of self-timed movements versus simple reactions. J Neurosci 26:2487-2498.

Martin JR (2003) Locomotor activity: a complex behavioural trait to unravel. Behav Process 64:145-160.

Martin JR (2004) A portrait of locomotor behaviour in Drosophila determined by a video-tracking paradigm. Behav Process 67:207-219.

Martin JR, Ernst R, Heisenberg M (1998) Mushroom bodies suppress locomotor activity in Drosophila melanogaster. Learn Mem 5:179-191.

Martin JR, Ernst R, Heisenberg M (1999a) Temporal pattern of locomotor activity in Drosophila melanogaster. J Comp Physiol A Neuroethol Sens Neural Behav Physiol 184:73-84.

Martin JR, Raabe T, Heisenberg M (1999b) Central complex substructures are required for the maintenance of locomotor activity in Drosophila melanogaster. J Comp Physiol A Neuroethol Sens Neural Behav Physiol 185:277-288.

Maye A, Hsieh CH, Sugihara G, Brembs B (2007) Order in spontaneous behavior. PLoS One 2:e443.

Pearson KG, Ramirez J (1997) Sensory modulation of pattern-generating circuits. In: Neurons, networks, and motor behavior (Stein PSG, Grillner S, Selverston AI, Stuart DG, eds), pp 225-235. Cambridge, MA: MIT.

Perrins R, Walford A, Roberts A (2002) Sensory activation and role of inhibitory reticulospinal neurons that stop swimming in hatching frog tadpoles. J Neurosci 22:4229-4240.

Purzner J, Paradiso GO, Cunic D, Saint-Cyr JA, Hoque T, Lozano AM, Lang AE, Moro E, Hodaie M, Mazzella F, Chen R (2007) Involvement of the basal ganglia and cerebellar motor pathways in the preparation of selfinitiated and externally triggered movements in humans. J Neurosci 27:6029-6036. 
Ramirez JM, Pearson KG (1988) Generation of motor patterns for walking and flight in motoneurones supplying bifunctional muscles in the locust. J Neurobiol 19:257-282.

Robertson RM (1986) Neuronal circuits controlling flight in the locust: central generation of the rhythm. Trends Neurosci 9:278-280.

Sandeman H, Sandeman R, Derby C, Schmidt M (1992) Morphology of the brain of crayfish, crabs, and spiny lobsters: a common nomenclature for homologous structures. Biol Bull 183:304-326.

Schall JD (2001) Neural basis of deciding, choosing and acting. Nat Rev Neurosci 2:33-42.

Schultz W (2000) Multiple reward signals in the brain. Nat Rev Neurosci 1:199-207.

Schultz W, Romo R (1992) Role of primate basal ganglia and frontal cortex in the internal generation of movements. I. Preparatory activity in the anterior striatum. Exp Brain Res 91:363-384.

Schultz W, Tremblay L, Hollerman JR, Mirenowicz J (1997) Delayed responding and reward signals: neurons coding component processes of goal-directed behavior in primate basal ganglia and orbitofrontal cortex.
In: The association cortex-structure and function (Sakata H, Mikami A, Fuster JM, eds). Amsterdam: Harwood.

Tanji J, Hoshi E (2008) Role of the lateral prefrontal cortex in executive behavioral control. Physiol Rev 88:37-57.

Tautz J, Müller-Tautz R (1983) Antennal neuropile in the brain of the crayfish: morphology of neurons. J Comp Neurol 218:415-425.

Tinbergen N (1951) The study of instinct. Clarendon: Oxford.

Venables WN, Ripley BD (2002) Modern applied statistics with S, Ed 4. New York: Springer.

Webb B (2004) Neural mechanisms for prediction: do insects have forward models? Trends Neurosci 27:278-282.

Wiersma CA (1958) On the functional connections of single units in the central nervous system of the crayfish, Procambarus clarkii Girard. J Comp Neurol 110:421-471.

Yamane S, Takahata M (2002) Experimental modification of stepping course in spontaneously initiated locomotor behavior in the crayfish Procambarus clarkii Girard. J Comp Physiol A Neuroethol Sens Neural Behav Physiol 188:13-23. 